OPEN ACCESS

Edited by:

Fabio Blandini,

Fondazione Istituto Neurologico Nazionale Casimiro Mondino (IRCCS), Italy

Reviewed by: Aaron Voigt,

Uniklinik RWTH Aachen, Germany

Kah-Leong Lim,

National Neuroscience Institute,

Singapore

*Correspondence:

Lori M. Buhlman

Ibuhlm@midwestern.edu

tThese authors have contributed equally to this work.

Received: 28 November 2017

Accepted: 31 January 2018

Published: 15 February 2018

Citation:

Cackovic J, Gutierrez-Luke S, Call GB, Juba A, O'Brien S, Jun $\mathrm{CH}$ and Buhlman LM (2018) Vulnerable Parkin Loss-of-Function Drosophila

Dopaminergic Neurons Have

Advanced Mitochondrial Aging,

Mitochondrial Network Loss and

Transiently Reduced Autophagosome Recruitment.

Front. Cell. Neurosci. 12:39. doi: 10.3389/fncel.2018.00039

\section{Vulnerable Parkin Loss-of-Function Drosophila Dopaminergic Neurons Have Advanced Mitochondrial Aging, Mitochondrial Network Loss and Transiently Reduced Autophagosome Recruitment}

\author{
Juliana Cackovic ${ }^{1 \dagger}$, Susana Gutierrez-Luke ${ }^{1 \dagger}$, Gerald B. Call ${ }^{1}$, Amber Juba ${ }^{2}$, \\ Stephanie O'Brien ${ }^{1}$, Charles H. Jun ${ }^{1}$ and Lori M. Buhlman ${ }^{2 *}$
}

${ }^{1}$ Arizona College of Medicine, Midwestern University, Glendale, AZ, United States, ${ }^{2}$ Department of Biomedical Sciences, College of Health Sciences, Midwestern University, Glendale, AZ, United States

Selective degeneration of substantia nigra dopaminergic (DA) neurons is a hallmark pathology of familial Parkinson's disease (PD). While the mechanism of degeneration is elusive, abnormalities in mitochondrial function and turnover are strongly implicated. An Autosomal Recessive-Juvenile Parkinsonism (AR-JP) Drosophila melanogaster model exhibits DA neurodegeneration as well as aberrant mitochondrial dynamics and function. Disruptions in mitophagy have been observed in parkin loss-of-function models, and changes in mitochondrial respiration have been reported in patient fibroblasts. Whether loss of parkin causes selective DA neurodegeneration in vivo as a result of lost or decreased mitophagy is unknown. This study employs the use of fluorescent constructs expressed in Drosophila DA neurons that are functionally homologous to those of the mammalian substantia nigra. We provide evidence that degenerating DA neurons in parkin loss-of-function mutant flies have advanced mitochondrial aging, and that mitochondrial networks are fragmented and contain swollen organelles. We also found that mitophagy initiation is decreased in park (Drosophila parkin/PARK2 ortholog) homozygous mutants, but autophagosome formation is unaffected, and mitochondrial network volumes are decreased. As the fly ages, autophagosome recruitment becomes similar to control, while mitochondria continue to show signs of damage, and climbing deficits persist. Interestingly, aberrant mitochondrial morphology, aging and mitophagy initiation were not observed in DA neurons that do not degenerate.

\footnotetext{
Abbreviations: atg8a, autophagy 8a; DA, dopaminergic; GABARAP, gamma amino butyric acid receptor associated protein; LC3, light chain 3; LNF, lane normalization factor; mfn2, mitofusin 2; mitoGFP, mitochondrially-targeted green fluorescent protein; park+/+, control park Drosophila; park+/-, heterozygous park loss-of-function Drosophila; park-/-, homozygous park loss-of-function Drosophila; PBS, phosphate-buffered saline; PBT, PBS containing 0.3 percent Triton $\mathrm{X}-100$; PD, Parkinson's disease; PE, post eclosion; PPL1, protocerebral posterior lateral region 1; ROC, receiver operating characteristic; TH, tyrosine hydroxylase; TOM, outer mitochondrial membrane translocase; UAS, upstream activating sequence; VDAC, voltage-dependent anion channel.
} 


\begin{abstract}
Our results suggest that parkin is important for mitochondrial homeostasis in vulnerable Drosophila DA neurons, and that loss of parkin-mediated mitophagy may play a role in degeneration of relevant DA neurons or motor deficits in this model.
\end{abstract}

Keywords: parkin, neurodegeneration, dopaminergic neurons, Parkinson's disease, autophagy, mitochondria, mitochondrial dynamics, MitoTimer

\section{INTRODUCTION}

A growing body of evidence suggests that parkin, an E3 ubiquitin ligase, plays an important role in mitochondrial integrity. The means by which parkin supports mitochondrial function are less clear. A widely held theory behind the mechanism by which loss of parkin function causes neurodegeneration is that parkin-deficient cells lack the ability, or have impaired ability, to rid themselves of depolarized mitochondria. The increasing presence of damaged mitochondria becomes an insurmountable burden that causes cells to undergo apoptosis (reviewed in Pickrell and Youle, 2015). The highly oxidizing mitochondrial environment warrants efficient mitochondrial turnover to maintain optimal cellular respiration, particularly in cells with high energy demands. Cytosolic parkin can be recruited to depolarized mitochondria in order to initiate mitophagy (Narendra et al., 2008; Hämäläinen et al., 2013). Parkinmediated ubiquitination of a variety of outer mitochondrial membrane substrates precedes autophagosomal engulfment of the mitochondrion. These ubiquitination events may serve as signals for autophagosome recruitment (Ding et al., 2010; Geisler et al., 2010; Narendra et al., 2010; Chan et al., 2011; Sarraf et al., 2013; Wong and Holzbaur, 2014; Lazarou et al., 2015). Whether the absence of parkin causes DA neurodegeneration as a result of lost or decreased mitophagy in vivo, however, is still unknown. Much of the evidence addressing the role of parkin in mitophagy comes from manipulations of overexpression systems in cultured cells, which facilitate elegant live imaging studies. Unlike neurons, immortalized cells rely on glycolysis, rather than oxidative phosphorylation as their main source of ATP (Crabtree, 1929). Drosophila park (Drosophila parkin/PARK2 ortholog) lossof-function mutants have neuron and muscle degeneration, defective spermatids and dysfunctional mitochondria that have aberrant morphology (Greene et al., 2003; Burman et al., 2012). Interestingly, park heterozygous loss-of function flies have motor deficits, but their DA neurons do not degenerate (Chambers et al., 2013). Park homozygous mutants that lack the glutathione S-transferase S1 (GstS1) gene (encoding antioxidant glutathione s-transferase) have more pronounced phenotypes, and GstS1 overexpression prevents DA neuron degeneration (Greene et al., 2005; Whitworth et al., 2005). This suggests that increased reactive oxygen species (ROS) load is a major factor in neurodegeneration caused by the absence of parkin. Indeed, advanced aging is regularly associated with increased ROS load (Beckman and Ames, 1998; Jacob et al., 2013). How the absence of parkin yields increased oxidative stress is unclear, but decreased mitophagy and/or proteasomemediated turnover of mitochondrial proteins may be implicated (Vincow et al., 2013).
We have explored the effects of parkin loss-of-function on mitochondrial network integrity, mitochondrial aging, autophagosome formation and mitophagy initiation in park mutant Drosophila (park ${ }^{25}$ ) (Greene et al., 2003) protocerebral posterior lateral region 1 (PPL1) and protocerebral posterior medial region 3 (PPM3) DA neurons. These neurons are functionally homologous to those of the mammalian substantia nigra pars compacta (reviewed in Strausfeld and Hirth, 2013); however, only PPL1 neurons degenerate in park loss-offunction flies (Whitworth et al., 2005). PPL1 neurons can be spared when autophagosome-promoting factor autophagy 8a (atg8a) is exogenously expressed (Burman et al., 2012). In order to address whether autophagosome formation and mitophagy initiation are affected by the park mutation, we utilized flies that express autophagosome marker mCherryatg8a (mCherry-atg8a; Ichimura et al., 2000; Nezis et al., 2009; Shpilka et al., 2011). We generated park mutant flies that express a mitochondrially targeted green fluorescent protein (mitoGFP) to measure mitochondrial network integrity (Rizzuto et al., 1995). Mitochondrial aging was observed using park mutant flies that express the MitoTimer construct, which encodes a mitochondrially-targeted DsRed variant. MitoTimer excitation and emission spectra shift from green to red as mitochondria age, likely as a result of oxidation at the Tyr-67 residue (Yarbrough et al., 2001; Laker et al., 2014). Increased levels of red MitoTimer also have been used to indicate decreases in mitochondrial turnover (Ferree et al., 2013; Hernandez et al., 2013). Using this in vivo model, we provide evidence that degenerating DA neurons may be vulnerable because of selective dependence on parkin-mediated mitophagy.

\section{MATERIALS AND METHODS}

\section{Drosophila Maintenance and Strains}

All flies were maintained on standard cornmeal molasses food at $25^{\circ} \mathrm{C}$ and $12 / 12$-h light/dark cycle. Flies were transferred to vials containing new food every 3-4 days. The park ${ }^{25}$ lossof-function mutants (Greene et al., 2003) were a gift from Leo Pallanck at the University of Washington in Seattle. $W^{1118}$-containing control stocks and Drosophila harboring tyrosine hydroxylase (TH)-GAL4 (P\{ple-GAL4.F\}; stock 8848), upstream activating sequence (UAS)-mitoGFP ( $P\{U A S$-mito-HAGFP.AP\}3; stock 8442), UAS-MitoTimer (stock 57323), and UASAtg8a ( $P\{U A S p$ - $m$ Cherry-Atg8a\}; stock 37750) constructs were obtained from the Bloomington Drosophila Stock Center at Indiana University, Bloomington (Rizzuto et al., 1995; FriggiGrelin et al., 2003; Nezis et al., 2009; Laker et al., 2014). All experimental flies were outcrossed to a $w^{1118}$ control line. 
The park $^{25}$ mutant genotypes were confirmed via PCR and/or via observation of park ${ }^{25}$ loss-of-function phenotype. Flies harboring UAS-fluorescent constructs were crossed with those harboring $\mathrm{TH}$-GAL4 in order to drive expression exclusively into TH-producing cells.

\section{Confirmation of park ${ }^{25}$ Alleles Using Polymerase Chain Reaction}

Presence and absence of the park ${ }^{25}$ allele was detected using a standard PCR protocol incorporating primers park25F ( $5^{\prime}$-GAT TGG CAA CAC TGA AGC- $\left.3^{\prime}\right)$ and park25R (5'-CTT TAC CAT CCC CCA ATC AA- $3^{\prime}$ ) designed in the Pallanck laboratory at the University of Washington, Seattle, WA, USA. Wild-type park product is $2.3 \mathrm{~kb}$, and the mutant park ${ }^{25}$ product is $960 \mathrm{bp}$. The $2.3 \mathrm{~kb}$ wild-type product is not amplified consistently; therefore, park(5) (5'-GAT ACG ACG GGA TTA CAA GGG G-3') and park(6) (5'-TGT CTT CTA GTA GCA ATG TGA CTT-3') primers were used to amplify a $272 \mathrm{bp}$ product that is absent from flies harboring the park ${ }^{25}$ allele.

\section{Immunohistochemistry}

Fly heads were removed, and brains were dissected on days 5, 10 and 20 post eclosion (PE: the day Drosophila emerge from their pupa cases) in phosphate-buffered saline (PBS). Brains were immediately fixed in $3.7 \%$ formaldehyde (VWR, West Chester, PA, USA) in PBS for $30 \mathrm{~min}$. Following four washes in 0.3\% PBT (PBS containing 0.3\% Triton X-100; MP Biomedical, Salon, OH, USA) for $5 \mathrm{~min}$, samples were placed in blocking solution (10\% normal goat serum [Sigma Aldrich, St. Louis, MO, USA] in $0.3 \%$ PBT) for $30 \mathrm{~min}$. Brains were probed with an anti-TH antibody raised in rabbit (EMD Millipore, Temecula, CA, USA) and diluted 1:100 in blocking solution overnight $(12-18 \mathrm{~h})$ at $4^{\circ} \mathrm{C}$. The next day, brains were washed fourusing a confocal microscope (Leica Microsyst times in $0.3 \%$ PBT for $5 \mathrm{~min}$. Samples were placed in blocking solution for $30 \mathrm{~min}$ before incubation with goat anti-rabbit $\operatorname{IgG}(\mathrm{H}+\mathrm{L})$ secondary antibody conjugated to Alexa Fluor 405 (Life Technologies, Grand Island, NY, USA) diluted 1:200 in blocking solution for a minimum of $2 \mathrm{~h}$ at room temperature (RT) to overnight at $4^{\circ} \mathrm{C}$. The brains were washed four times in PBS for $5 \mathrm{~min}$, then they were mounted on microscope slides using Vectashield mounting medium (Fisher Scientific, Pittsburgh, PA, USA) for MitoTimer and mitoGFP-only brains or Prolong Diamond mounting medium (Life Technologies, Eugene, OR, USA) for brains containing mCherry-atg8a and mitoGFP. Slides were coded so that the experimenter was blind to genotype and age during image capture and processing.

\section{Image Capture}

Z-stacks of one PPL1 and one PPM3 cell cluster per brain was captured using a confocal microscope (Leica Microsystems, Buffalo Grove, IL, USA) at $630 \times$ with 1.5 zoom (optical section thickness of $1.039 \mu \mathrm{m}$ and a z-step size of $0.34 \mu \mathrm{m}$ ). A $405 \mathrm{~nm}$ laser was used to excite the Alexa-405 secondary antibody conjugate to visualize TH. Both GFP (excitation range, 475-495 nm/emission, 520-560 nm), and the green variant of MitoTimer (peak excitation/emission, 488/518 nm) were excited with a $488 \mathrm{~nm}$ laser. A $532 \mathrm{~nm}$ laser was used to excite the red variant of MitoTimer, which has an excitation peak of approximately $558 \mathrm{~nm}$ and an emission maximum at $583 \mathrm{~nm}$, and mCherry, whose excitation maximum is $587 \mathrm{~nm}$ and emission maximum is $610 \mathrm{~nm}$. Each image was captured at a frequency of $400 \mathrm{~Hz}$.

For each fluorophore, gain and offset remained constant when capturing images (e.g., Gain was set at 800 and offset at -1.0 for all green MitoTimer images). In order to account for inconsistencies in immunolabeling and mitoGFP levels in measurements of mitochondrial object size, sphericity and mitochondrial network volume per cell, laser intensity was adjusted, and a pseudo-colored channel indicating overexposure was used so that images could be captured when about $2 \%$ of the image was overexposed. For MitoTimer and atg8a protocols, laser intensity was fixed for each fluorophore.

\section{Digital Image Data Collection}

Image Pro Premier 3D (Media Cybernetics Inc., Rockville, MD, USA) was used to measure mitochondrial sphericity, volumes and/or colocalization for all fluorophores in control $\left(\right.$ park $\left.^{+/+}\right)$, park heterozygous ( park $^{+/ 25}$, referred to as, "park ${ }^{+/-}$” in text)

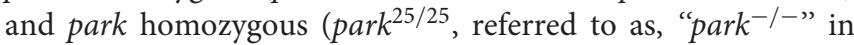
text) flies.

\section{Mitochondrial Sphericity and Object Volume Measurements}

An automatic intensity threshold for one PPL1 and one PPM3 cluster per brain was obtained for park park $^{+/}$, par (PPL1 only) and park $^{-/-}$flies expressing the mitoGFP fluorophore construct under the control of TH-GAL4 ( $w^{1118}$; UAS-mitoGFP/+; TH-GAL4/+ for park ${ }^{+/+}, w^{1118} ;$ UASmitoGFP/+; TH-GAL4, park ${ }^{25} /+$ for park ${ }^{+/-}$, and $w^{1118} ;+{ }^{w 1118}$; TH-GAL4, park ${ }^{25}$ /UAS-mitoGFP, park ${ }^{25}$ for park $^{-/-}$), and 3D structures of mitochondrial objects in the GFP channel with fluorescence intensity above threshold were selected. Objects outside of the TH-labeled area were removed so that all objects used for data collection were found within PPL1 DA somas and proximal projections. To determine whether mitochondrial network fragmentation occurs in park mutant flies, we measured volume and the inverse of sphericity (six times object volume divided by the object's diameter surface, or $[6 \mathrm{~V}] / \mathrm{DS}$ ) for each mitochondrial object. The average mitochondrial object volume and inverse of sphericity for each cluster was recorded for at least 10 clusters per condition. PPL1 mitochondrion size frequency distribution histograms were generated by placing objects into small, medium and large bins based on volume. Bin size was determined by taking the difference of the largest and smallest data point and dividing it by three. Large mitochondrion sphericity analyses include the sphericity data for the largest five percent of all PPL1 mitochondrial objects.

\section{MitoTimer Volume Measurements}

Three dimensional objects of mitochondrial MitoTimer proteins in the GFP and DSred channels above a standardized intensity threshold were selected from one PPL1 and one PPM3 cluster 
per brain in park ${ }^{+/+}$, park pal- $^{+-}$and park $^{-/-}$flies expressing MitoTimer under the control of TH-GAL4 $\left(w^{1118} ;+{ }^{w 1118}\right.$; UAS-MitoTimer/TH-GAL4, for park ${ }^{+/+} ; w^{1118} ;+{ }^{w 1118}$; UASMitoTimer, park ${ }^{25} /$ TH-GAL4 for park ${ }^{+/-}$; and $w^{1118} ;+^{w 1118}$; UAS-MitoTimer, park ${ }^{25} /$ TH-GAL4, park $^{25}$ for park pl- $^{-}$). GFPand DSred- positive structures outside the TH-labeled region were removed so that all structures used were found within DA somas and proximal projections. Total volume for each channel was obtained by calculating the sum of the volume measurements of individual 3D mitochondrial objects within the TH-labeled regions of one brain cluster. Ratios of total red to total green volume per brain also were calculated (Laker et al., 2014) for at least 11 PPL1 clusters per condition.

\section{Autophagosome Formation}

In order to determine the relative levels of autophagosome formation, red objects fluorescing above background and between $0.1 \mathrm{~nm}^{3}$ and $1 \mu \mathrm{m}^{3}$ were counted to represent structures of mCherry-atg8a puncta from one PPL1 and one PPM3 (data not shown) region in park $^{+/+}$, park pal- $^{+/}$and park $^{-/-}$flies expressing mCherry-atg8a (atg8a, autophagosome marker) and mitoGFP under the control of TH-GAL4 $\left(w^{1118} ; \quad U A S-m C h e r r y A t g 8 a, \quad U A S-m i t o G F P /+; \quad T H-G A L 4 /+\right.$ for park ${ }^{+/+}, w^{1118}$; UAS-mCherryAtg8a, UAS-mitoGFP/+; THGAL4, park ${ }^{25} /+$ for park ${ }^{+/-}$, and $w^{1118}$; UAS-mCherryAtg8a, UAS-mitoGFP/+; TH-GAL4 park ${ }^{25} /$ park $^{25}$ for park $^{-/-}$). We established a standardized method of measuring red objects representing autophagosomes based on qualitative observations of staining patterns. The number of atg8a-positive autophagosomes within TH-labeled somas was divided by the number of TH-positive neurons for at least night brain clusters per condition.

\section{PPL1 Dopaminergic Neuron Number}

The number of TH-positive neurons in one PPL1 cluster per brain was determined using the Image Pro Premier software manual counting tool to count the number of TH-labeled cells in individual z-stack frames for at least nine PPL1 clusters per condition. The counting tool adds a digital tag that remains on the screen as the observer moves from frame to frame to avoid duplicate counts. TH-positive cells were counted in park $^{+/+}$, park par- $^{+/}$and park pl- $^{-/}$flies expressing mCherryatg8a and mitoGFP under the control of TH-GAL4 (Genotype information is provided above).

\section{Autophagosome Recruitment to Mitochondria}

We used Image Pro Premier 3D co-localization tool to count the number of mCherry-positive objects (identified as described above) that also were labeled with GFP (mitoGFP) as indicated by a positive Pearson's correlation coefficient. The number of co-localization events within one TH-labeled PPL1 and one PPM3cluster per brain is reported for at least five clusters for park $^{+/+}$, park pal- $^{+/}$and park $^{-/-}$flies expressing mCherry-atg8a and mitoGFP (genotypes described above).

\section{Mitochondrial Network Volume Per Cell}

We took the sum of volumes for mitoGFP-positive objects selected for measurements of mitochondrial object volume for each TH-labeled cell within one PPL1 and one PPM3 cluster per brain and divided that value by the corresponding soma volume measurement (indicated by TH-labeling). TH-positive neurons with clearly-defined, $\mathrm{TH}$ signal were included the analysis. Data represent at least 15 PPL1 and six PPM3 clusters in park $^{+/+}$, park pal- $^{+/}$and park ${ }^{-/-}$flies expressing mitoGFP under the control of TH-GAL4 ( $w^{1118}$; UAS-mitoGFP/+; TH-GAL4/+ for park ${ }^{+/+}, w^{1118}$; UAS-mitoGFP/+; TH-GAL4, park ${ }^{25} /+$ for park $^{+/-}$, and $w^{1118} ;+{ }^{w 1118} ;$ TH-GAL4, park ${ }^{25} /$ UAS-mitoGFP, park $^{25}$ for park ${ }^{-/-}$).

\section{Western Blotting}

Approximately 200-300 park $^{+/+}$, park $^{+/-}$and park $^{-/-}$flies $\left(w^{1118}, w^{1118} ;\right.$ park $^{25} /+$, and $w^{1118} ;$ park $^{25} /$ park $\left.^{25}\right)$ aged day 1 through $14 \mathrm{PE}$ were transferred to $50 \mathrm{ml}$ conical tubes. Each tube subsequently was frozen in liquid nitrogen and briskly shaken to separate heads from bodies. Frozen heads and bodies were transferred to a no 25 sieve (Fisher Scientific, Waltham, MA, USA) that had been chilled to $-80^{\circ} \mathrm{C}$. Collected heads were ground thoroughly using a dounce homogenizer (Fisher Scientific, Waltham, MA, USA) containing $200 \mu \mathrm{l}$ ice-cold fractionation buffer $(250 \mathrm{mM}$ Sucrose, $10 \mathrm{mM}$ Tris $\mathrm{pH} 7.4,0.15 \mathrm{mM} \mathrm{MgCl}_{2}$ ) freshly supplemented with protease inhibitor cocktail (Roche, Indianapolis, IN, USA). Extracts were centrifuged at $1000 \times g$ for $5 \mathrm{~min}$ at $4^{\circ} \mathrm{C}$. Supernatants were centrifuged again at $1000 \times g$ for $5 \mathrm{~min}$ at $4^{\circ} \mathrm{C}$. Brain-enriched supernatants were collected, and total protein was determined using the BCA method (Pierce Waltham, MA, USA). Samples were reduced, and $10 \mu \mathrm{g}$ of protein was separated in $12 \%$ Bis-Tris SDS-PAGE gels (Bio-Rad, Hercules, CA, USA) and transferred to a $0.45 \mu \mathrm{m}$ nitrocellulose membrane (Bio-Rad). The membrane was treated with REVERT $^{\mathrm{TM}}$ Total Protein Stain following the recommended protocol (LICOR, Lincoln, NE, USA) and imaged using a $700 \mathrm{~nm}$ laser on an Odyssey infra-red imaging system (LICOR). The membrane was then briefly rinsed with Milli-Q ${ }^{\circledR}$ water and blocked under slight agitation in blocking solution (5\% non-fat dry milk diluted in Tris-buffered saline with $2 \%$ Tween 20 [TBST]) for at least $1 \mathrm{~h}$ at RT. In order to identify atg8a, the Drosophila gamma amino butyric acid receptor associated protein (GABARAP) ortholog, the membrane was probed with a rabbit monoclonal anti-GABARAP primary antibody (ab109364 lot\#GR154272-6; Abcam, Cambridge, MA, USA) diluted 1:10,000 in blocking solution overnight at $4^{\circ} \mathrm{C}$ under slight agitation. The membrane was washed five times with TBST under moderate agitation at RT and probed with an infrared fluorescent goat-anti-rabbit secondary antibody (IRDye ${ }^{\circledR} 800$ CW; LICOR, lot\#C50602-05) diluted 1:10,000 in blocking solution for at least $1 \mathrm{~h}$ under slight agitation at RT. The membrane was washed five times with TBST under moderate agitation at RT followed by one wash in Tris-buffered saline. Washed membranes were allowed to dry in the dark and were subsequently imaged with an $800 \mathrm{~nm}$ laser using the Odyssey imaging system.

Membrane images were analyzed using Image Studio Version 4.0 (LICOR). The total protein approach for normalization was used, as evidence suggests commonly used reference proteins can be differentially expressed (Eaton et al., 2013). REVERT ${ }^{\mathrm{TM}}$ 


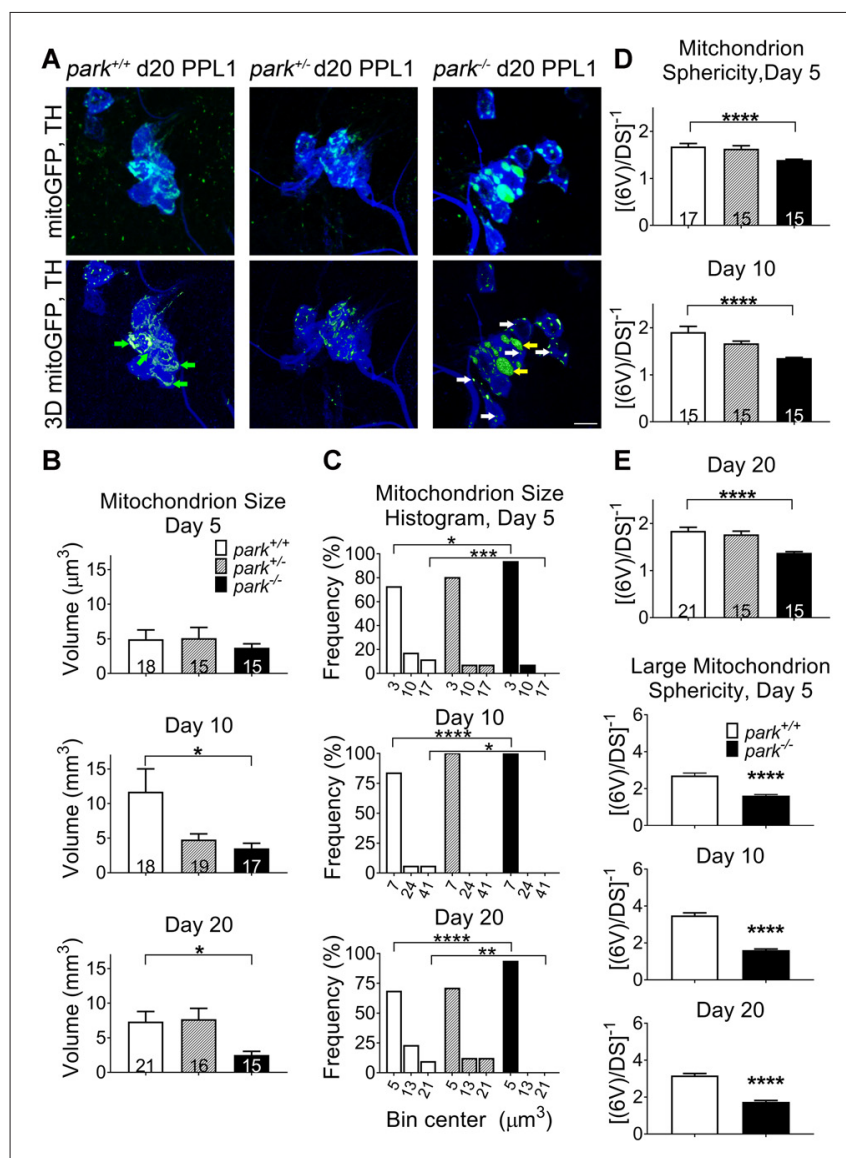

FIGURE 1 | Parkin loss-of-function flies have increased mitochondrial fragmentation and swelling in protocerebral posterior lateral region 1 (PPL1) DA neurons. Brains of park mutant flies expressing the mitochondrially targeted green fluorescent protein (mitoGFP) construct in tyrosine hydroxylase (TH)-producing cells were dissected, stained for TH (blue), and fixed on days 5,10 or 20 post eclosion (PE). Images in (A) are representative intensity sums of Z-stacks used as 3D projections for the park control (park ${ }^{+/+}$),

heterozygote (park ${ }^{+/-}$) and homozygote (park ${ }^{-/-}$) conditions on day 20. Green (mitoGFP) labeling in the bottom row of $\mathbf{( A )}$ represents 3D isosurfaces that are generated based on signal above an automatic bright threshold. Digital image enhancement steps were standardized for green fluorophores. After using standardized image capture parameters, we used Image Pro Premier 3D image analysis software to measure and categorize the mitochondrial object size (B) and inverse of sphericity ([6V]/DS) (D) of mitoGFP objects within the $\mathrm{TH}$-labeled regions for one PPL1 region per brain. Mitochondrion size frequency distribution histograms were generated by placing objects into small, medium and large bins based on volume (C). Bin size was determined by taking the difference of the largest and smallest data point and dividing it by three. The angled numbers along the $\mathrm{x}$-axis indicate the bin center, or the value that falls halfway between the smallest and largest values of its bin. We also measured the inverse of sphericity for the largest five percent of objects (E). Green arrows indicate large, interconnected (not swollen) mitochondria. White and yellow arrows indicate fragmented and large, swollen mitochondria, respectively. Numbers in histogram bars indicate the number of PPL1 regions analyzed. For $* P<0.05$; for $* * P<0.01$; for ${ }^{* * *} P<0.001$; for ${ }^{* * * * *} P<0.0001$. Error bars represent standard error of the mean; scale bar represents five microns.

staining of proteins within the $37-75 \mathrm{kDa}$ range was selected as the total protein signal for each lane (Eaton et al., 2013). A lane normalization factor (LNF) was calculated by dividing the
REVERT $^{\mathrm{TM}}$ signal for each lane by the REVERT ${ }^{\mathrm{TM}}$ signal for the lane that had the highest signal. Atg8a bands at approximately $14 \mathrm{kDa}$ and $12 \mathrm{kDa}$ represent uncleaved and cleaved (activated) forms of atg8a, respectively, and a LNF was calculated for the 14 and $12 \mathrm{kDa}$ bands. Finally, the cleaved or uncleaved LNF for each band was divided by the total protein LNF from the corresponding lane to determine the normalized signal. Total (uncleaved plus cleaved) atg8a and the percent of cleaved atg8a were calculated. Each data point is an average signal of three replicates of the same sample. Samples from four collection days were analyzed.

\section{Climbing Assays}

Climbing (negative geotaxis) assays were performed by placing day 5,10 or 20 days PE park ${ }^{+/+}$, park par $^{+/}$and park $k^{+/-}$ flies expressing mitoGFP and mCherry-atg8a under the control of TH-GAL4 (genotypes described above) into one of 16 transparent polycarbonate tubes $(5 \mathrm{~mm}$ diameter, $80 \mathrm{~mm}$ length, one fly per tube). A MutiBeam Activity Monitor (TriKinetics Inc. Waltham, MA, USA) held tubes in the vertical position so that 17 independent infrared beams could pass through each tube; the distance between the first and last beam is $51 \mathrm{~mm}$. White yarn was placed just inside of the top and bottom of the polycarbonate tubes in order to trap the fly in the infrared detection zone. Data for five to eight flies per genotype were collected simultaneously, and control flies ( $w^{1118}$; UASmCherryAtg8a, UAS-mitoGFP/+; TH-GAL4/+) were included in all climbing trials. Each time a fly crosses an infrared beam, a count is recorded, allowing for determination of the fly's position in the tube each second for 20 min. "Height climbed" is the distance in mm of a flies' continuous trajectory from one position to a higher position in the tube. Each time a fly climbs up again after moving downward, a new "height climbed" is recorded. The total height climbed during the 20 -min recording period was measured, and divided by number of climbs to calculate average height climbed. A receiver operating characteristic (ROC) curve was generated from mitoGFP, mCherry-atg8a control fly climbing data to distinguish "climbing" from "nonclimbing" flies (Microsoft Excel; Microsoft, Redmond, WA, USA). Climbing data for 6-50 flies per condition is reported.

\section{Statistical Analyses}

To determine the effect of the park mutation on mitochondrial network morphology and volume, atg8a protein levels, and number of autophagosomes, dopaminergic (DA) neurons and co-localization events, comparisons to control were completed via an ordinary one-way ANOVA followed by Dunnett's multiple comparisons test when data were normally distributed. For cases in which data did not pass the Shapiro-Wilk normality test, comparisons to control were made using Kruskal-Wallis tests followed by Dunn's multiple comparisons test. Unpaired $t$-tests were performed to determine the effect of genotype on the sphericity of large mitochondrial objects in PPL1. To determine effects of the park mutation and on day Parkinson's disease

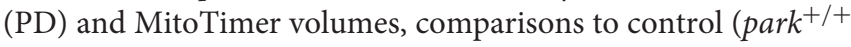
and day 5, respectively) were made via a two-way ANOVA followed by Dunnet's multiple comparisons test. Fisher's exact 
tests were used to determine the effect of the park mutation on the frequency distribution of large and small mitochondrial objects. Fisher's exact tests were also used to determine the distributions of climbing and non-climbing park heterozygous and homozygous mutant Drosophila compared to that of control, to one another, and within genotypes to measure the effect of time. A Mann-Whitney test comparing ranks was performed to determine the effect of the homozygous park mutation on the number of mCherry-atg8a objects colocalized with mitoGFP per cell. For all tests, $\alpha=0.05$.

\section{RESULTS}

\section{Vulnerable Dopaminergic Neuron Mitochondria Are Selectively Fragmented and Swollen in Parkin Loss-of-Function \\ Flies}

Since mitochondrial network fragmentation and swelling can be a sign of damage, we measured the size and sphericity of PPL1 and PPM3 DA neuron mitochondria in control flies $\left(\right.$ park $\left.^{+/+}\right)$, park heterozygotes (park ${ }^{+/}$, PPL1 only) and park homozygotes $\left(\right.$ park $\left.^{-/}\right)$that also express mitoGFP and TH-GAL4. In contrast to the interconnected, ribbon-shaped mitochondria observed in control (green arrows), most park $^{-/-}$ PPL1 mitochondria appeared to be fragmented (Figure 1A, white arrows). A small proportion of large, round mitochondrial objects was observed in the park ${ }^{-/-}$condition (Figure 1A, yellow arrows). These apparently swollen mitochondria were few in number relative to fragmented mitochondria; thus, the average mitochondrial volume per PPL1 region was smaller in the park $^{-/-}$condition on days 10 and 20 (Figures 1A-C). Mitochondria were also more spherical on days 10 and 20, suggesting that park ${ }^{-/-}$PPL1 mitochondrial networks are generally fragmented (Figures 1A,D). The largest five percent of $\mathrm{park}^{-/-}$mitochondria were more spherical, indicating that they are swollen, rather than interconnected (Figure 1E). Thus, park p $^{-/}$PPL1 mitochondrial networks are generally fragmented, which can indicate damage and preparation for mitophagy. These mitochondria also show signs of swelling, which not only indicates damage, but also impeded mitophagy. There was no effect of the heterozygous park mutation on object sphericity or volume (Figure 1). Interestingly, mitochondrial fragmentation and swelling were not observed in park $^{-/-}$PPM3 neurons, which do not degenerate (Figure 2).

\section{Mitochondrial Aging Is Advanced in Parkin Mutant PPL1 but Not PPM3 DA Neurons}

Mitochondrial damage and network fragmentation can be caused by uncontrolled levels of macromolecule oxidation. We found that park $^{-/-}$flies expressing MitoTimer and TH-GAL4 had greater volumes of red, aged MitoTimer per PPL1 cluster than park $^{+/+}$flies on day 5, 10 and day 20 PE (Figures 3A,D). There were no changes in levels of green (newly synthesized) MitoTimer, and the ratio of red to green total volume per cluster was increased on days 10 and 20 (Figures 3B-D).

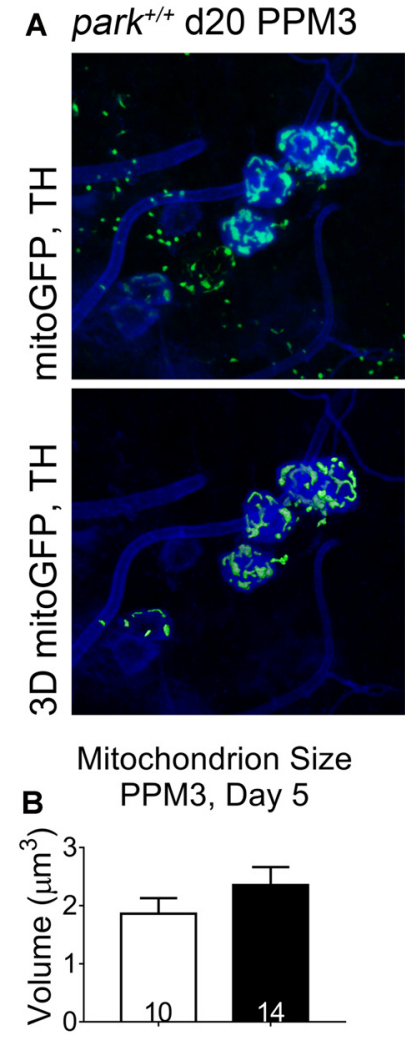

Day 10

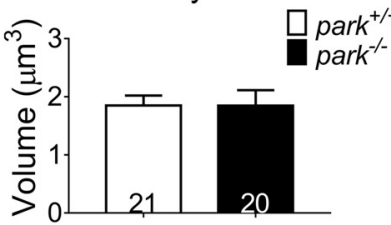

Day 20

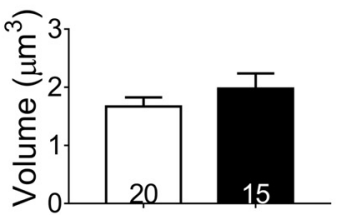

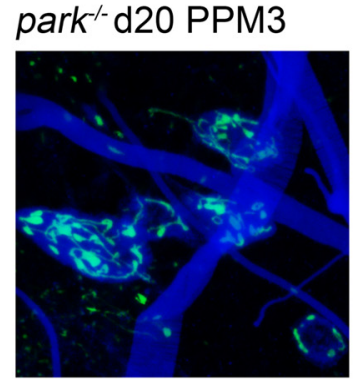

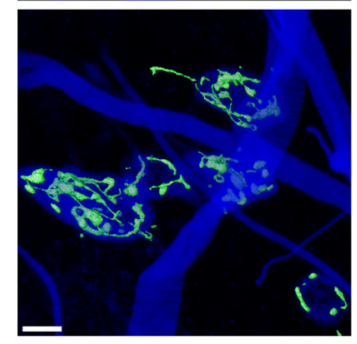

Mitchondrion Sphericity C PPM3, Day 5

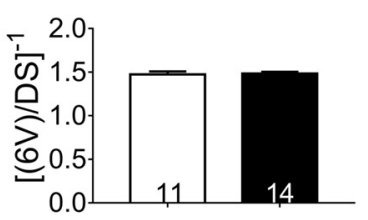

Day 10

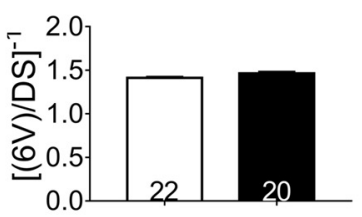

Day 20

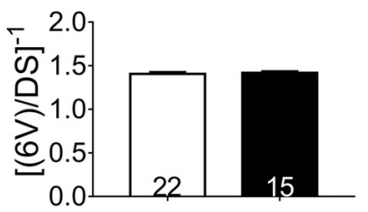

FIGURE 2 | Non-degenerating DA neurons in parkin loss-of-function flies do not have fragmented or swollen mitochondria networks. Brains of park mutant flies expressing the mitoGFP construct in TH-producing cells were dissected, stained for TH (blue), and fixed on days 5, 10 or 20 PE. Images in (A) are representative intensity sums of Z-stacks used as $3 \mathrm{D}$ projections for the park $^{+/+}$and park ${ }^{-1-}$ conditions on day 20. Green (mitoGFP) labeling in the bottom row of $\mathbf{( A )}$ represents 3D isosurfaces that are generated based on signal above an automatic bright threshold. Digital image enhancement steps were standardized for green fluorophores. After using standardized image capture parameters, we used Image Pro Premier 3D image analysis software to measure and categorize the mitochondrial object size $\mathbf{( B )}$ and inverse of sphericity ([6V]/DS) (C) of mitoGFP objects within the TH-labeled regions for one PPM3 region per brain. Numbers in histogram bars indicate the number of regions analyzed. Error bars represent standard error of the mean; scale bar represents five microns.

There was no effect of day PE on MitoTimer measurements, indicating that as flies age, mitochondrial turnover is consistent. 


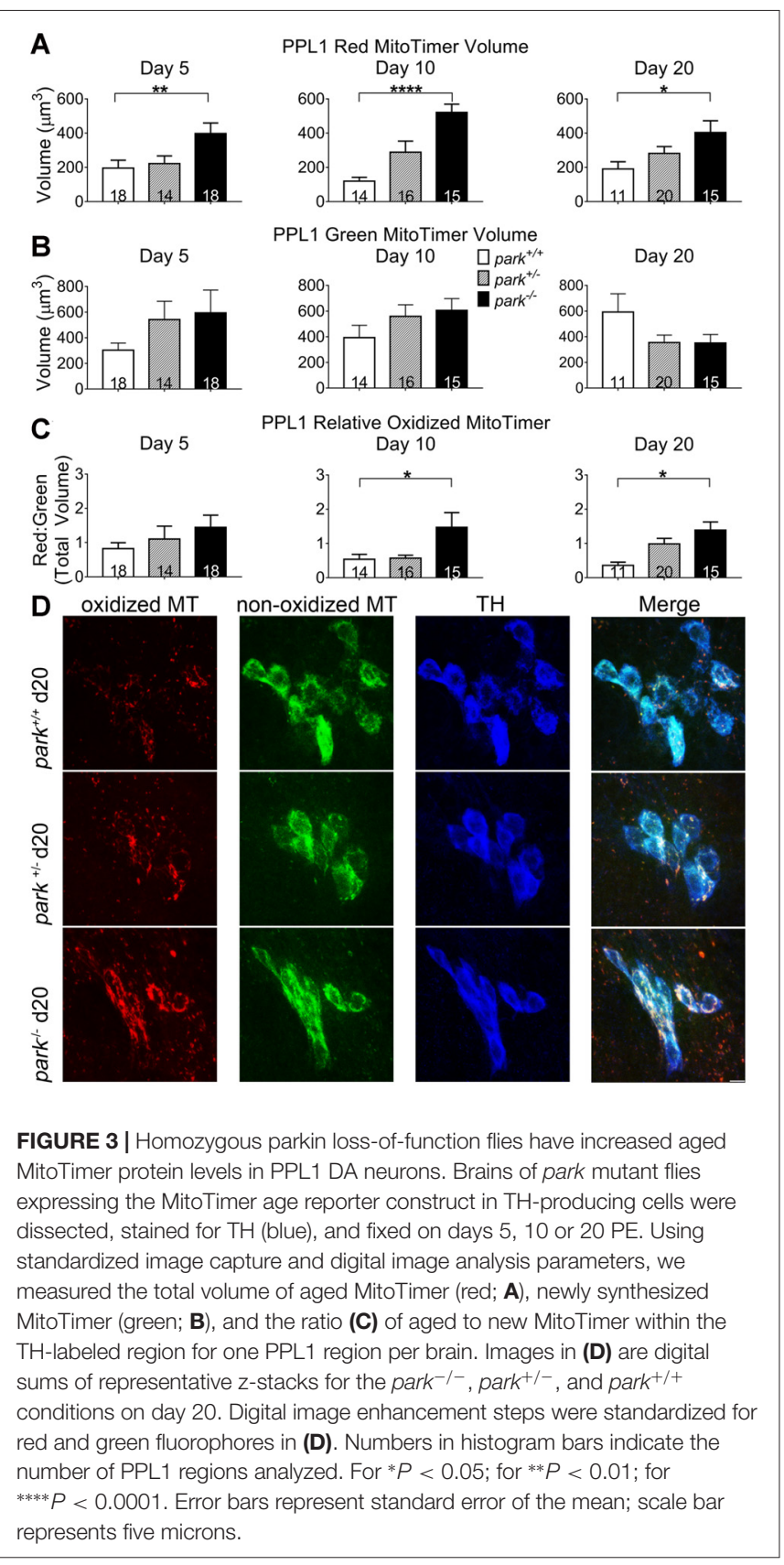

Levels of red MitoTimer in PPM3 were unaffected by the park mutation, suggesting that only susceptible DA neurons show signs of aberrant mitochondrial aging (Figure 4). Thus, accelerated mitochondrial aging, likely caused by increased mitochondrial protein oxidation, may contribute to the park mutant PPL1 degeneration.

\section{Autophagosome-Promoting atg8a Activation and Autophagosome Numbers Are Not Affected by the park Mutation}

To facilitate autophagosome formation, atg8a is cleaved at the C-terminal before it is conjugated to phosphatidylethanolamine

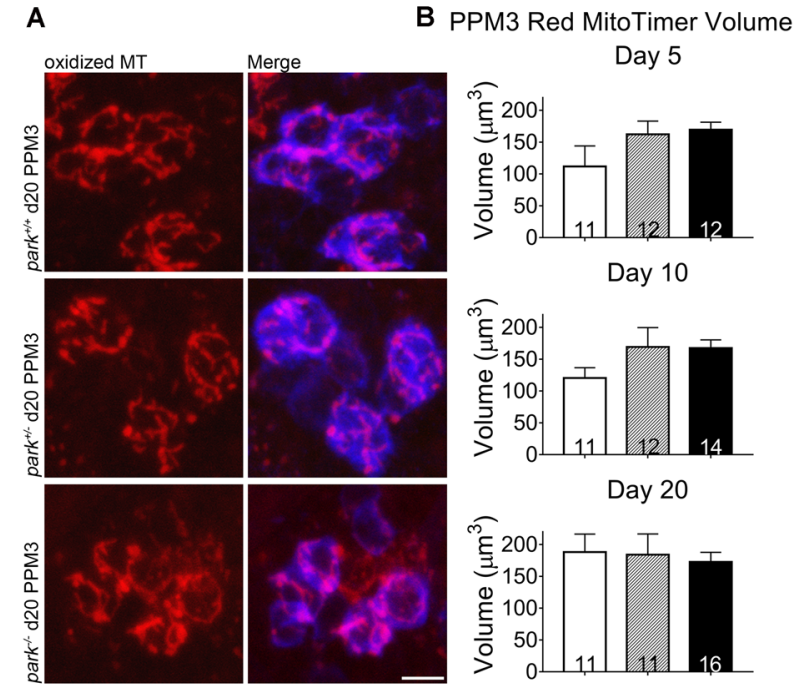

FIGURE 4 | Homozygous parkin loss-of-function non-degenerating DA neurons show signs of normal mitochondrial aging. Brains of park mutant flies expressing the MitoTimer age reporter construct in $\mathrm{TH}$-producing cells were dissected, stained for TH (blue), and fixed on days 5, 10 or 20 PE. Using standardized image capture and digital image analysis parameters, we measured the total volume of aged MitoTimer (red) within the $\mathrm{TH}$-labeled region for one PPM3 region per brain $\mathbf{( A , B ) . ~ I m a g e s ~ i n ~} \mathbf{( A )}$ are digital sums of representative $z$-stacks for the park $^{-/-}$, park ${ }^{+/-}$, and park ${ }^{+/+}$conditions on day 20. Digital image enhancement steps were standardized for red fluorophores. Numbers in histogram bars indicate the number of PPM3 regions analyzed. Error bars represent standard error of the mean; scale bar represents five microns.

of pre-autophagic vesicles (Kirisako et al., 2000). In cell lines that express little or no parkin, autophagosome formation is unaffected by exogenous parkin (Ding et al., 2010). In order to address whether disruptions in autophagosome formation occur in an in vivo parkin loss-of-function model, brainenriched fractions from day 1 to $14 \mathrm{PE} \mathrm{park}^{+/+}, \mathrm{park}^{+/-}$and park $^{-/}$flies were used for western blotting. We observed no effect of genotype on total atg8a or percent activated relative to total (Figure 5). In order to address whether disruptions in autophagosome formation may be limited to susceptible neurons, we counted autophagosomes in park mutant flies that express mCherry-atg8a and TH-GAL4. Similar numbers of mCherry-atg8a positive autophagosomes per cell (PPL1) or per cluster (PPM3; data not shown) were detected in all three genotypes (Figures 6A,B). Thus, parkin does not appear to play a role in Drosophila brain atg8a activation, or PPL1/PPM3 DA neuron autophagosome formation.

\section{Promotion of Autophagy Initiation Fails to Prevent PPL1 Dopaminergic Neurodegeneration and Climbing Behavior Deficits in park Mutants}

Studies have shown that exogenous expression of autophagosome-forming atg8a can prevent DA neuron mitochondrial membrane depolarization and degeneration 


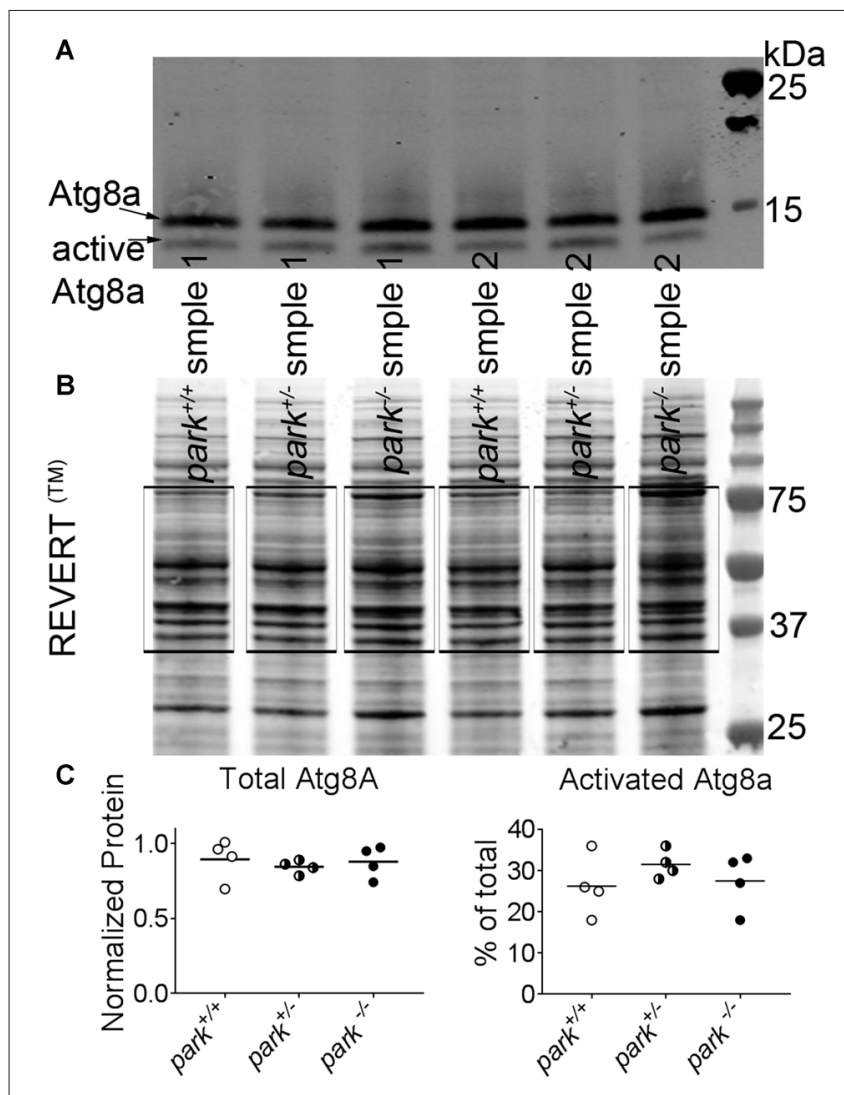

FIGURE 5 | Levels of active and inactive autophagy 8 a (atg8a) are unchanged in park mutant fly heads. Ten microgram of homogenized park park $^{-1-}$, or park $^{+/+}$fly brain fractions per lane was transferred to a nitrocellulose membrane, which was incubated in REVERT TM protein stain and imaged using an automatic bright threshold. The REVERT ${ }^{\mathrm{TM}}$ stain was washed, and the membrane was probed with a rabbit anti-gamma amino butyric acid receptor associated protein (GABARAP) primary antibody (recognizing Drosophila atg8a) followed by an anti-rabbit secondary antibody with an infrared fluorescent conjugate (IRDye ${ }^{\circledR} 800 \mathrm{CW}$, LICOR) (A). Total protein was determined by measuring REVERT ${ }^{\mathrm{TM}}$ fluorescence between $75 \mathrm{kDa}$ and $37 \mathrm{k}$ Da for each lane (B, black boxes). Fluorescence signal for each lane was divided by the signal for the lane that had the highest intensity. For atg8a measurements, the signals from the inactive $14 \mathrm{kDa}$ and the active $12 \mathrm{kDa}$ bands were divided by the corresponding signal for the band that had the highest intensity. To control for loading, these values were divided by the relative REVERT ${ }^{\mathrm{TM}}$ total protein value for each lane (Eaton et al., 2013). There was no effect of the park mutation on total or activated atg8a levels (C). Each data point is an average of three technical replicates for one collection day; lysates were generated on four different collection days (indicated in histogram bars).

in flies aged to at least day 15 PE (Burman et al., 2012). TH-driven mCherry-atg8a expression does not prevent DA neuron degeneration in park $^{-/-}$flies (Figure 6C). Negative geotaxis is a motivated behavior that is facilitated in part by DA neurons (Riemensperger et al., 2011; Barone and Bohmann, 2013). Deficiencies in climbing behavior have been detected in park homozygous and heterozygous mutants (Greene et al., 2003; Chambers et al., 2013), and these deficiencies persisted in park ${ }^{+/-}$flies on days 10 and 20, and in park par $^{-/}$flies on days 5, 10 and 20 (Figure 6D). Homozygous park mutants had decreased climbing proficiency compared to park heterozygotes on days 5 and 20. Climbing deficits worsened from day 5 to day 10 in park pal- $^{+/}$and from day 10 to 20 in park par $^{-/}$(Figure 6D). Thus, the exogenous expression of mCherry-atg8a in vulnerable DA neurons fails to rescue to the park mutant phenotype.

\section{Mitophagy Initiation Is Selectively and Transiently Decreased in Homozygous park Mutant PPL1 DA Neurons}

As an initiating step toward mitophagy, autophagosomes are recruited to damaged mitochondria. Whether parkin is required for autophagosome recruitment seems to depend on the experimental model and method (Narendra et al., 2008; Ding et al., 2010; Allen et al., 2013; Strappazzon et al., 2015). In park mutant flies expressing mCherry-atg8a, mitoGFP and $\mathrm{TH}$ GAL4, we found that the number of mCherry puncta colocalizing with GFP was decreased in park ${ }^{-/-}$PPL1 DA clusters on days 5 and 10 PE (Figures 7A,B). Interestingly, autophagosome recruitment in park $^{-/-}$was similar to that of park $^{+/+}$on day 20 , suggesting that compensatory mechanisms for parkinindependent autophagosome recruitment are triggered after day 10 , as the phenotype worsens. Fewer DA neurons were detected on day 20, so we measured the number of colocalized objects per cell and again found no difference (Figure 7C). Harboring one functional copy of the park gene was sufficient for maintaining control levels of autophagosome recruitment, and recruitment was unaffected in PPM3 (Figure 7D). Thus, parkin appears to play a role in, but is not required for, mitophagy initiation in vulnerable PPL1 neurons. It does not seem to be involved in autophagosome recruitment for non-degenerating DA neurons. These data suggest that decreased autophagosome recruitment may be a primary contributor to park mutant PPL1 degeneration.

\section{Decreased Mitophagy Initiation in PPL1 DA Neurons Does Not Cause an Increase in Mitochondrial Network Volume}

Because autophagosome recruitment (mitophagy induction) is decreased in park homozygotes, we hypothesized that mitochondrial network volume would be increased in park ${ }^{-/-}$ flies. However, we found that mitochondrial network volume per cell was similar in mitoGFP and TH-GAL4-expressing park $^{+/+}$and park ${ }^{-/-}$on days 5 and 20. Intriguingly, PPL1 mitochondrial network volume was decreased in park ${ }^{-/-}$ on day 10 (Figures 8A,B), when autophagosome recruitment was decreased, suggesting that alternative forms of mitochondrial turnover are initiated. PPL1 mitochondrial network volume was decreased in park $^{+/-}$on days 10 and 20 (Figure 8B). Thus, signs of mitochondrial damage are evident in heterozygote PPL1 but not PPM3 neurons. Mitochondrial network volume was decreased in park p $^{-1-}$ PPM3 on day 10, and it recovers on day 20 (Figure 8C).

\section{DISCUSSION}

We have shown that mitochondria in vulnerable DA neurons selectively harbor signs of damage in an in vivo parkin lossof-function model. Our experiments were performed on 

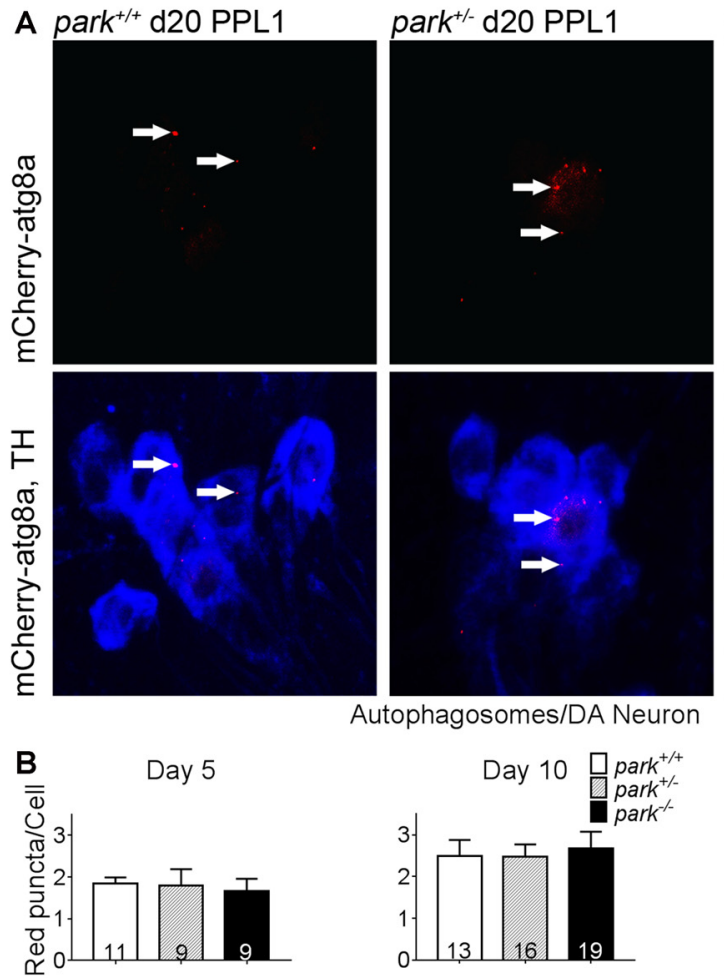

C Day 5

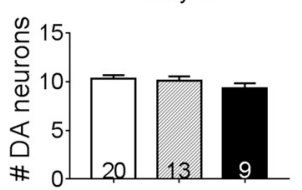

park $^{/-}$d20 PPL1
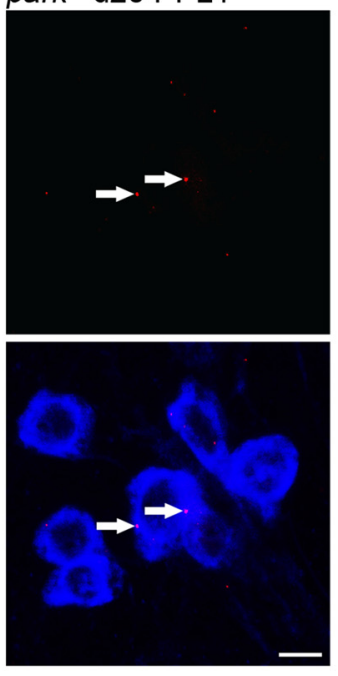

Day 20

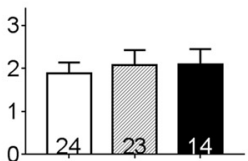

Day 20

PPL1 DA Neuron Survival Day 10

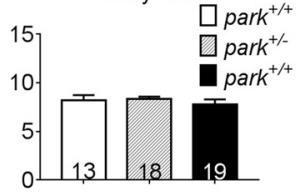

D

Climbing, mCherry-atg8a Day 5
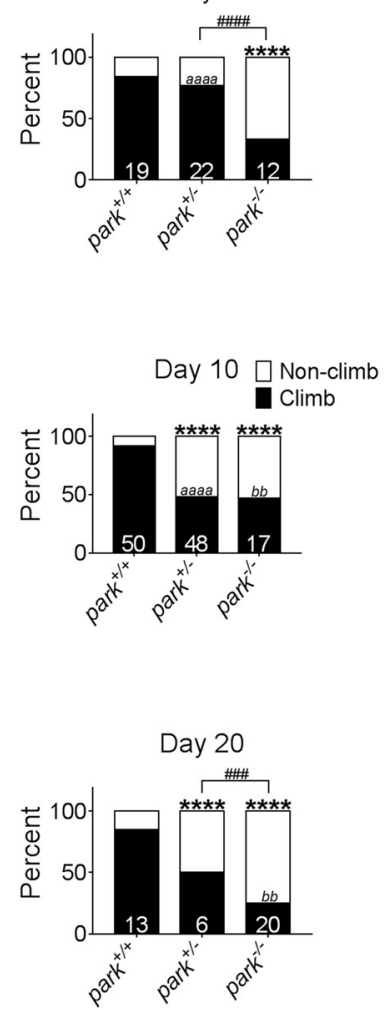

FIGURE 6 | Exogenous expression of atg8a in TH-producing neurons does not ameliorate DA degeneration or climbing deficits in park mutant flies. Brains of flies expressing the mitoGFP and mCherry-atg8a constructs in TH-producing cells were dissected, stained for TH (blue), and fixed on days 5 , 10 or 20 PE. Images in (A) are digital sums of representative $z$-stacks for day 20 park $^{+/+}$, park ${ }^{+/-}$, park ${ }^{-/-}$PPL1; digital image enhancement steps were standardized for the mCherry (red) fluorophore. Using standardized image capture and digital image analysis parameters, we identified and counted the number of atg8a puncta (white arrows) within TH-labeled regions and divided that value by the number of TH-positive cells for one PPL1 region per brain (B). We also counted the number of TH-positive cells per cluster (C). (D) Climbing assays were performed by placing individual flies expressing mitoGFP and mCherry-atg8a into polycarbonate tubes; the fly's position in the tube was recorded each second for $20 \mathrm{~min}$. The total distance climbed during the 20-min recording period was measured, and divided by number of climbs to calculate the average height climbed. A receiver operating characteristic (ROC) curve was generated from mitoGFP, mCherry-atg8a control fly climbing data to distinguish "climbing" from "non-climbing" flies. Numbers in histogram bars indicate the number of PPL1 regions analyzed for (B,C); for (D), number in histogram bars represent the number of flies tested. For ${ }^{* *}$ and ${ }^{\text {bb }} P<0.01$; for ${ }^{\# \# \#} P<0.001$; ${ }^{\# \# \# \#<0.0001 \text {; for }}{ }^{* * * *}$ and ${ }^{\text {aaaa }} P<0.0001$. Error bars represent standard error of the mean; scale bar represents five microns.

days 5,10 and $20 \mathrm{PE}$ in order to determine whether signs of mitochondrial damage and disruptions in mitophagy are associated with park mutant phenotypes (Greene et al., 2003; Chambers et al., 2013). Park P $^{-/}$PPL1 but not PPM3 DA neurons have fragmented mitochondrial networks, swollen mitochondria and accelerated mitochondrial aging, which could result from mitochondrial protein oxidation. Inability to maintain homeostatic mitochondrial respiration and/or ROS levels could result from decreased proteasome- or lysosomemediated turnover of mitochondrial proteins (Lim et al., 2005; Whitworth et al., 2005; Mortiboys et al., 2008; Burman et al., 2012; Vincow et al., 2013). The park mutation does not affect atg8a activation in the fly brain or autophagosome number in DA neurons, and exogenous expression of mCherryatg8a in DA neurons does not prevent PPL1 degeneration or motivated behavior. Mitophagy initiation is unaffected in non-degenerating DA neurons, but it is transiently decreased in park $^{-/-}$PPL1. Our data suggest that parkin's role in autophagosome recruitment differs among DA regions, and that decreased mitochondrial recruitment and accelerated aging may be principle causes of neurodegeneration in the absence of parkin. Recovery of autophagosome recruitment implies that alternative mechanisms for mitophagy initiation are triggered as mitochondrial pathology and deficits in motivated behavior persist. Mitochondrial network volumes are decreased in park mutants, even when mitophagy 


\section{A mitoGFP}
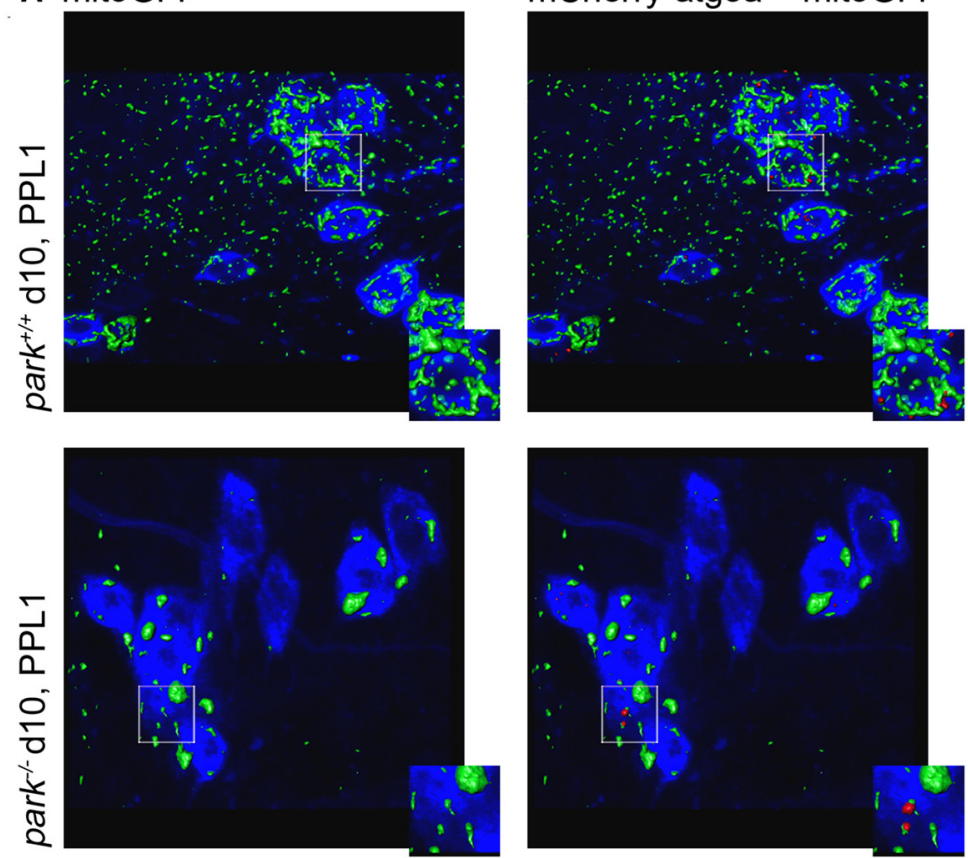

PPL1 Autophagosome Recruitment

B

Day 5

Day 10

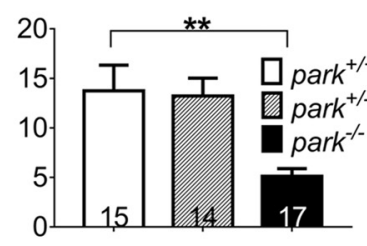

20
15
10
5
0

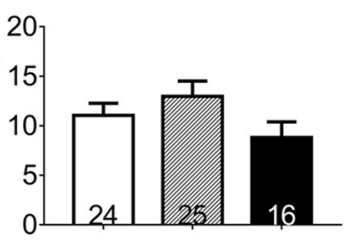

Day 20

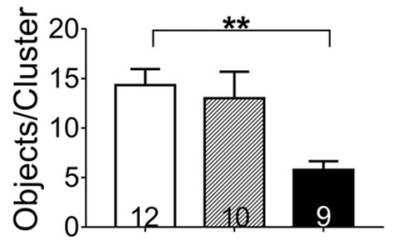

PPM3 Autophagosome Recruitment

D

Day 5

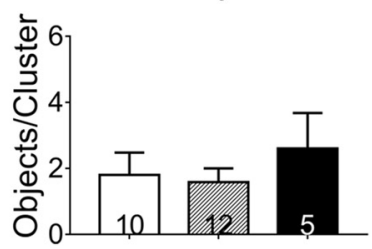

Day 10

Day 20
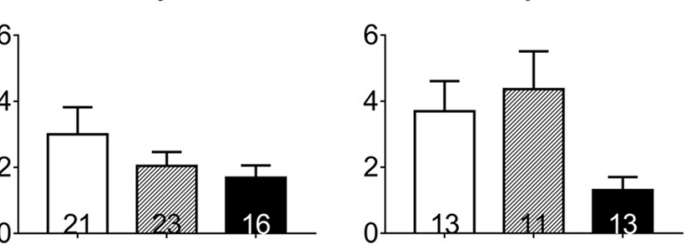
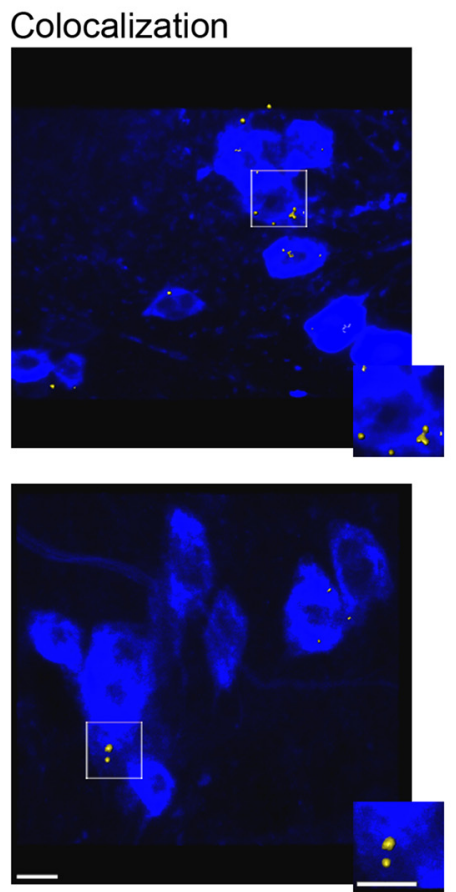

C

PPL1 Autophagosome Recruitment per Cell Day 20

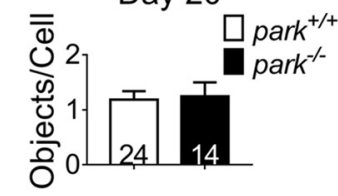

FIGURE 7 | Autophagosome recruitment to mitochondria is selectively and transiently reduced in park mutant PPL1. Brains of park mutant flies expressing the mitoGFP and mCherry-atg8a constructs in TH-producing cells were dissected, stained for TH (blue), and fixed on days 5 , 10 or 20 PE. Red and green objects in (A)

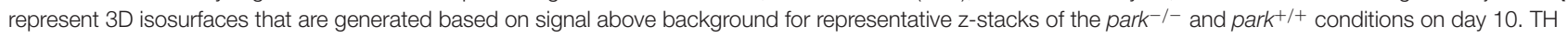
signal is represented as a summary of the z-stack. Insets highlight mitochondria that are colocalized with atg8a (A). Using standardized image capture and digital image processing analysis, we identified and counted the number of atg8a puncta that colocalized with mitoGFP according to a positive Pearson's correlation coefficient within the TH-labeled regions for one PPL1 and one PPM3 region per brain (B,D, respectively). Because there was a decrease in PPL1 neurons in park ${ }^{-1-}$ flies and no change in colocalizations per cell on day 20, we divided the number of colocalizations by the number of TH-positive (DA) neurons for the corresponding PPL1 cluster in day 20 park $^{+/+}$and park ${ }^{-1-}$ (C). Numbers in histogram bars indicate the number of DA regions analyzed. For ** $P<0.01$. Error bars represent standard error of the mean; scale bars represent five microns.

initiation is decreased, suggesting that alternative forms of mitochondrial turnover may be involved. PPL1 neurons may be selectively vulnerable because they harbor damaged mitochondria in a system where mitophagy initiation is impaired.
Parkin can promote turnover of protein substrates by directing them to lysosomes via Lysine 63 ubiquitination (DossPepe et al., 2005; Lim et al., 2005; Olzmann et al., 2007; Gegg et al., 2010; Poole et al., 2010; Ziviani et al., 2010; Chan et al., 2011; Glauser et al., 2011; Rakovic et al., 2011). Our data 

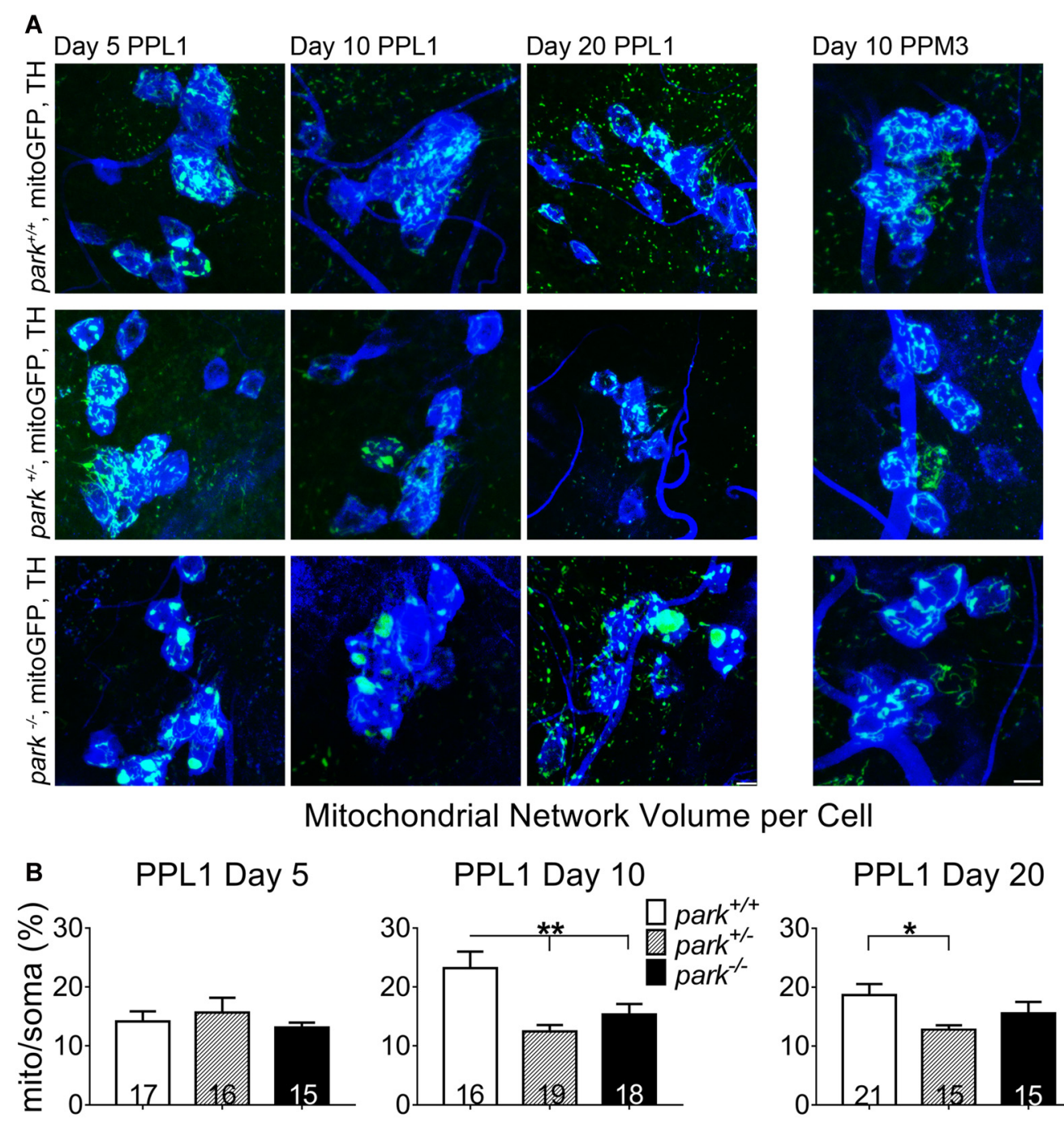

PPL1 Day 20
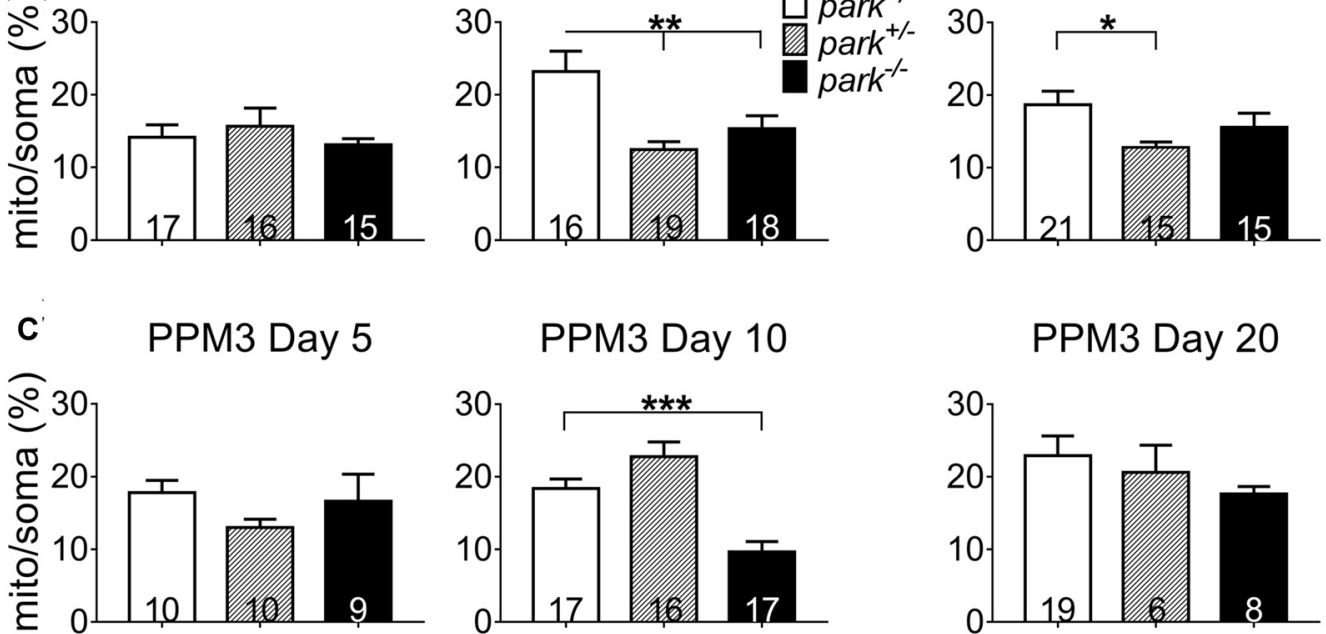

PPM3 Day 10

PPM3 Day 20
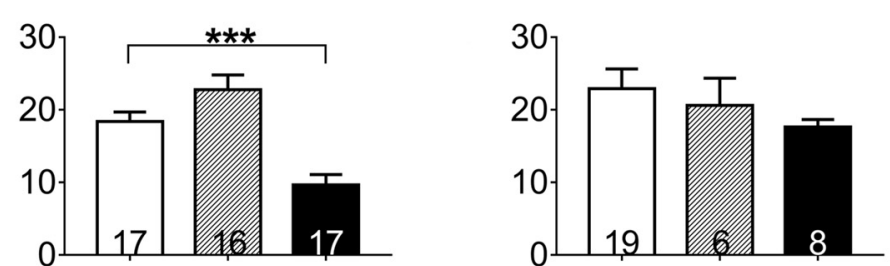

FIGURE 8 | Mitochondrial network volume is decreased in park mutant fly DA cells. Brains of park mutant flies expressing only the mitoGFP construct in TH-producing cells were dissected, stained for TH (blue), and fixed on days 5, 10 or 20 PE. Using standardized image capture and digital image analysis, we took the sum of the volume of all mitoGFP objects fluorescing above an automatically generated brightness threshold that were within the TH-labeled region for one cell, and divided that value by the volume of the corresponding TH-labeled soma and proximal projections for one region per brain (B,C). Images in (A) are sums of representative $\mathrm{Z}$-stacks for park ${ }^{+/+}$, park ${ }^{+/-}$and park ${ }^{-/-}$day 5, 10 and 20 PPL1 and day 10 PPM3. Digital image enhancement steps were standardized for green fluorophores. Numbers in histogram bars indicate the number of regions analyzed. For $* P<0.05$; for ${ }^{* *} P<0.01$; for ${ }^{* * *} P<0.001$. Error bars represent standard error of the mean; scale bar represents five microns.

show for the first time that parkin plays a non-essential role in autophagosome recruitment to mitochondria in susceptible DA neurons in vivo, and that parkin is not required for autophagosome recruitment in non-degenerating DA neurons.
Interestingly, mitochondrial network volume is decreased when autophagosome recruitment is decreased or unaffected, and levels of green, newly synthesized MitoTimer are steady. This suggests that an autophagosome-independent method of 
mitochondrial turnover may be at work (Vincow et al., 2013). Future experiments could determine whether PINK1 levels or activity are augmented to recover PPL1 autophagosome recruitment in the absence of parkin (Lazarou et al., 2015). Since homozygote PPL1 neurons degenerate by day 20 , it may be that surviving DA neurons are more robust, and/or have more efficient mitochondrial turnover. Nonetheless, PPL1 neurons that are present on day 20 show signs of mitochondrial damage. Systemic exogenous expression of atg8a previously has been shown to restore mitochondrial membrane potential, prevent PPL1 DA neuron loss, improve lifespan, and promote resistance to oxidative stress and the accumulation of ubiquitinated proteins in Drosophila (Simonsen et al., 2008; Burman et al., 2012). We detected no change in atg8a activation, and a small, perhaps delayed decrease in PPL1 DA neuron number in park homozygotes compared to control flies expressing TH-driven mCherry-atg8a. The question remains whether exogenous atg8a expression could reduce mitochondrial fragmentation or relative red MitoTimer ratios in park PPL1. We observed no change in mitophagy initiation in park ${ }^{-/-}$PPM3 or in park ${ }^{+/-}$PPL1, neither of which degenerate. Yet $p^{2} k^{+/-}$PPL1 mitochondria show signs of dysfunction at time points when motor deficits occur (Chambers et al., 2013). Thus, degeneration of susceptible Drosophila dopaminergic neurons may result from a combination of decreased parkin-mediated mitophagy and premature mitochondrial aging induced by oxidative stress. In Drosophila, parkin has been shown to facilitate autophagosome-independent respiratory chain enzyme subunit turnover; however, whether these proteins are targeted to the proteasome or delivered to the lysosome in mitochondriaderived vesicles is unclear (Soubannier et al., 2012; Vincow et al., 2013). Parkin facilitates proteasome-mediated turnover of inner membrane morphology and respiratory chain enzyme function stabilizer myeloid cell leukemia 1 (MCL-1), pro-apoptotic factor, Bax, and translocase of the outer membrane (TOM) subunits (Chan et al., 2011; Yoshii et al., 2011; Johnson et al., 2012; Perciavalle et al., 2012; Charan et al., 2014). The TOM complex must function at optimal capacity in order to maintain mitochondrial functions, since all but 13 mitochondrially-expressed proteins must be imported. Whether disruption in TOM subunit turnover contributes to age acceleration and/or fragmentation of mitochondria as observed here remains to be elucidated. Parkin can promote mitochondrial network connectivity, perhaps by facilitating turnover of mitochondrial fission protein drp1 and its outer membrane receptor, fis1 (Lutz et al., 2009; Wang H. et al., 2011). On the other hand, exogenous parkin expression can promote mitochondrial fragmentation (Ziviani et al., 2010; Buhlman et al., 2014), and the Drosophila parkin mutant phenotype improves with over-expression of drp1 or decreased expression of fusion proteins mitofusin 2 (mfn2) or opal (Deng et al., 2008; Poole et al., 2008). Parkin-overexpression may induce mitochondrial network fragmentation via K48 ubiquitination of outer membrane fusion proteins $\mathrm{mfn} 1$ and $\mathrm{mf} 2$ and/or microtubule associated motor protein, miro-1, a Rho GTPase that connects mitochondria to microtubules and facilitates their motility (Gegg et al., 2010; Poole et al., 2010; Ziviani et al., 2010; Chan et al., 2011; Glauser et al., 2011; Rakovic et al., 2011; Wang X. et al., 2011; Birsa et al., 2014; Kazlauskaite et al., 2014). Mitochondrial fusion can buffer the effects of minor damage to mitochondria (Rossignol et al., 2004; Tondera et al., 2009; Rambold et al., 2011), and fragmentation facilitates mitophagy when mitochondrial networks are damaged beyond repair. Cell-specific energy requirements may determine whether mitochondrial networks become interconnected or fragmented in the absence of parkin (Rafelski, 2013); they may also determine the demand for mitophagy.

\section{CONCLUSION}

The role of parkin in mitophagy and mitochondrial homeostasis in various cell types has been extensively described prior to this study. We have provided evidence that mitochondrial network fragmentation, age acceleration, and decreased mitophagy initiation may be directly implicated in DA neurodegeneration in vivo. Changes in mitophagy initiation are not associated with non-degenerating DA neurons. Thus, our data suggest that Drosophila PPL1 neurons are selectively dependent on parkin-mediated mitophagy. These vulnerable neurons appear to invoke compensation for the loss of parkin by triggering parkinindependent mitophagy and alternative forms of mitochondrial turnover. Critical differences in gene expression, energy demands and synaptic partners of PPL1 and PPM3 remain to be explored. It is of note that MitoTimer, mitoGFP and mCherry-atg8a object measurements were limited to those distributed in cell bodies and proximal projections. Mitochondrial morphology, respiration and turnover may differ among regions like soma, axons, axon terminals and dendrites (Chang et al., 2006; Ashrafi et al., 2014). Nonetheless, our results provide new insight on the effect of the park mutation on susceptible DA neuron mitochondria in vivo, and promote a better understanding of the mechanisms of degeneration caused by parkin loss-offunction.

\section{AUTHOR CONTRIBUTIONS}

JC was involved in protocol development for measurements of mitochondrial shape and volume, and she performed and analyzed data for Figures 1, 6. She also contributed to the writing of the manuscript. SG-L was involved in protocol development for MitoTimer and mCherry-Atg8 analyses; and performed and analyzed data for Figures 2, 4, 5, and contributed to writing the manuscript. GBC generated genetic crosses for Drosophila strains, provided critical consultation on project development and critically edited the manuscript. AJ performed western blotting experiments and analyzed western blotting data and also contributed to writing the manuscript. SO performed and analyzed climbing data for day 5 Drosophila. $\mathrm{CHJ}$ performed PCR to confirm park25 genotypes. LMB supervised the project, generated the figures and wrote the manuscript. 


\section{FUNDING}

This work was supported by Midwestern University College of Health Sciences and Arizona College of Medicine.

\section{REFERENCES}

Allen, G. F., Toth, R., James, J., and Ganley, I. G. (2013). Loss of iron triggers PINK1/Parkin-independent mitophagy. EMBO Rep. 14, 1127-1135. doi: 10.1038/embor.2013.168

Ashrafi, G., Schlehe, J. S., LaVoie, M. J., and Schwarz, T. L. (2014). Mitophagy of damaged mitochondria occurs locally in distal neuronal axons and requires PINK1 and Parkin. J. Cell Biol. 206, 655-670. doi: 10.1083/jcb.201 401070

Barone, M. C., and Bohmann, D. (2013). Assessing neurodegenerative phenotypes in Drosophila dopaminergic neurons by climbing assays and whole brain immunostaining. J. Vis. Exp. 74:e50339. doi: 10.3791/50339

Beckman, K. B., and Ames, B. N. (1998). The free radical theory of aging matures. Physiol. Rev. 78, 547-581. doi: 10.1152/physrev.1998.78.2.547

Birsa, N., Norkett, R., Wauer, T., Mevissen, T. E., Wu, H. C., Foltynie, T., et al. (2014). Lysine 27 ubiquitination of the mitochondrial transport protein Miro is dependent on serine 65 of the Parkin ubiquitin ligase. J. Biol. Chem. 289, 14569-14582. doi: $10.1074 /$ jbc.m114.563031

Buhlman, L., Damiano, M., Bertolin, G., Ferrando-Miguel, R., Lombès, A., Brice, A., et al. (2014). Functional interplay between Parkin and Drp1 in mitochondrial fission and clearance. Biochim. Biophys. Acta 1843, 2012-2026. doi: 10.1016/j.bbamcr.2014.05.012

Burman, J. L., Yu, S., Poole, A. C., Decal, R. B., and Pallanck, L. (2012). Analysis of neural subtypes reveals selective mitochondrial dysfunction in dopaminergic neurons from parkin mutants. Proc. Natl. Acad. Sci. U S A 109, 10438-10443. doi: 10.1073/pnas.1120688109

Chambers, R. P., Call, G. B., Meyer, D., Smith, J., Techau, J. A., Pearman, K., et al. (2013). Nicotine increases lifespan and rescues olfactory and motor deficits in a Drosophila model of Parkinson's disease. Behav. Brain Res. 253, 95-102. doi: 10.1016/j.bbr.2013.07.020

Chan, N. C., Salazar, A. M., Pham, A. H., Sweredoski, M. J., Kolawa, N. J., Graham, R. L., et al. (2011). Broad activation of the ubiquitin-proteasome system by Parkin is critical for mitophagy. Hum. Mol. Genet. 20, 1726-1737. doi: $10.1093 / \mathrm{hmg} / \mathrm{ddr} 048$

Chang, D. T., Honick, A. S., and Reynolds, I. J. (2006). Mitochondrial trafficking to synapses in cultured primary cortical neurons. J. Neurosci. 26, 7035-7045. doi: 10.1523/jneurosci.1012-06.2006

Charan, R. A., Johnson, B. N., Zaganelli, S., Nardozzi, J. D., and LaVoie, M. J. (2014). Inhibition of apoptotic Bax translocation to the mitochondria is a central function of parkin. Cell Death Dis. 5:e1313. doi: 10.1038/cddis.2014.278

Crabtree, H. G. (1929). Observations on the carbohydrate metabolism of tumours. Biochem. J. 23, 536-545. doi: 10.1042/bj0230536

Deng, H., Dodson, M. W., Huang, H., and Guo, M. (2008). The Parkinson's disease genes pink1 and parkin promote mitochondrial fission and/or inhibit fusion in Drosophila. Proc. Natl. Acad. Sci. U S A 105, 14503-14508. doi: 10.1073/pnas. 0803998105

Ding, W. X., Ni, H. M., Li, M., Liao, Y., Chen, X., Stolz, D. B., et al. (2010). Nix is critical to two distinct phases of mitophagy, reactive oxygen species-mediated autophagy induction and Parkin-ubiquitin-p62-mediated mitochondrial priming. J. Biol. Chem. 285, 27879-27890. doi: 10.1074/jbc. m110.119537

Doss-Pepe, E. W., Chen, L., and Madura, K. (2005). $\alpha$-synuclein and parkin contribute to the assembly of ubiquitin lysine 63-linked multiubiquitin chains. J. Biol. Chem. 280, 16619-16624. doi: 10.1074/jbc.m413 591200

Eaton, S. L., Roche, S. L., Hurtado, M. L., Oldknow, K. J., Farquharson, C., Gillingwater, T. H., et al. (2013). Total protein analysis as a reliable loading control for quantitative fluorescent Western blotting. PLoS One 8:e72457. doi: 10.1371/journal.pone. 0072457

Ferree, A. W., Trudeau, K., Zik, E., Benador, I. Y., Twig, G., Gottlieb, R. A., et al. (2013). MitoTimer probe reveals the impact of autophagy, fusion and motility

\section{ACKNOWLEDGMENTS}

We thank Giulia Bertolin at the Institute of Genetics and Development of Rennes and Mitra Esfandiarei at Midwestern University for critically reading this manuscript.

on subcellular distribution of young and old mitochondrial protein and on relative mitochondrial protein age. Autophagy 9, 1887-1896. doi: 10.4161/auto. 26503

Friggi-Grelin, F., Coulom, H., Meller, M., Gomez, D., Hirsh, J., and Birman, S. (2003). Targeted gene expression in Drosophila dopaminergic cells using regulatory sequences from tyrosine hydroxylase. J. Neurobiol. 54, 618-627. doi: 10.1002/neu.10185

Gegg, M. E., Cooper, J. M., Chau, K. Y., Rojo, M., Schapira, A. H., and Taanman, J. W. (2010). Mitofusin 1 and mitofusin 2 are ubiquitinated in a PINK1/parkin-dependent manner upon induction of mitophagy. Hum. Mol. Genet. 19, 4861-4870. doi: 10.1093/hmg/ddq419

Geisler, S., Holmström, K. M., Skujat, D., Fiesel, F. C., Rothfuss, O. C., Kahle, P. J., et al. (2010). PINK1/Parkin-mediated mitophagy is dependent on VDAC1 and p62/SQSTM1. Nat. Cell Biol. 12, 119-131. doi: 10.1038/ncb2012

Glauser, L., Sonnay, S., Stafa, K., and Moore, D. J. (2011). Parkin promotes the ubiquitination and degradation of the mitochondrial fusion factor mitofusin 1 J. Neurochem. 118, 636-645. doi: 10.1111/j.1471-4159.2011.07318.x

Greene, J. C., Whitworth, A. J., Andrews, L. A., Parker, T. J., and Pallanck, L. J. (2005). Genetic and genomic studies of Drosophila parkin mutants implicate oxidative stress and innate immune responses in pathogenesis. Hum. Mol. Genet. 14, 799-811. doi: 10.1093/hmg/ddi074

Greene, J. C., Whitworth, A. J., Kuo, I., Andrews, L. A., Feany, M. B., and Pallanck, L. J. (2003). Mitochondrial pathology and apoptotic muscle degeneration in Drosophila parkin mutants. Proc. Natl. Acad. Sci. U S A 100, 4078-4083. doi: 10.1073/pnas.0737556100

Hämäläinen, R. H., Manninen, T., Koivumäki, H., Kislin, M., Otonkoski, T., and Suomalainen, A. (2013). Tissue- and cell-type-specific manifestations of heteroplasmic mtDNA 3243A $>\mathrm{G}$ mutation in human induced pluripotent stem cell-derived disease model. Proc. Natl. Acad. Sci. U S A 110, E3622-E3630. doi: $10.1073 /$ pnas. 1311660110

Hernandez, G., Thornton, C., Stotland, A., Lui, D., Sin, J., Ramil, J., et al. (2013). MitoTimer: a novel tool for monitoring mitochondrial turnover. Autophagy 9 , 1852-1861. doi: 10.4161/auto.26501

Ichimura, Y., Kirisako, T., Takao, T., Satomi, Y., Shimonishi, Y., Ishihara, N. et al. (2000). A ubiquitin-like system mediates protein lipidation. Nature 408, 488-492. doi: $10.1038 / 35044114$

Jacob, K. D., Noren Hooten, N., Trzeciak, A. R., and Evans, M. K. (2013). Markers of oxidant stress that are clinically relevant in aging and age-related disease. Mech. Ageing Dev. 134, 139-157. doi: 10.1016/j.mad.2013.02.008

Johnson, B. N., Berger, A. K., Cortese, G. P., and Lavoie, M. J. (2012). The ubiquitin E3 ligase parkin regulates the proapoptotic function of Bax. Proc. Natl. Acad. Sci. U S A 109, 6283-6288. doi: 10.1073/pnas.1113248109

Kazlauskaite, A., Kondapalli, C., Gourlay, R., Campbell, D. G., Ritorto, M. S., Hofmann, K., et al. (2014). Parkin is activated by PINK1-dependent phosphorylation of ubiquitin at Ser65. Biochem. J. 460, 127-139. doi: 10.1042/BJ20140334

Kirisako, T., Ichimura, Y., Okada, H., Kabeya, Y., Mizushima, N., Yoshimori, T., et al. (2000). The reversible modification regulates the membrane-binding state of Apg8/Aut7 essential for autophagy and the cytoplasm to vacuole targeting pathway. J. Cell Biol. 151, 263-276. doi: 10.1083/jcb.151.2.263

Laker, R. C., Xu, P., Ryall, K. A., Sujkowski, A., Kenwood, B. M., Chain, K. H., et al. (2014). A novel MitoTimer reporter gene for mitochondrial content, structure, stress and damage in vivo. J. Biol. Chem. 289, 12005-12015. doi: 10.1074/jbc. M113.530527

Lazarou, M., Sliter, D. A., Kane, L. A., Sarraf, S. A., Wang, C., Burman, J. L., et al. (2015). The ubiquitin kinase PINK1 recruits autophagy receptors to induce mitophagy. Nature 524, 309-314. doi: 10.1038/nature14893

Lim, K. L., Chew, K. C., Tan, J. M., Wang, C., Chung, K. K., Zhang, Y., et al. (2005). Parkin mediates nonclassical, proteasomal-independent ubiquitination of synphilin-1: implications for Lewy body formation. J. Neurosci. 25, 2002-2009. doi: 10.1523/jneurosci.4474-04.2005 
Lutz, A. K., Exner, N., Fett, M. E., Schlehe, J. S., Kloos, K., Lämmermann, K., et al. (2009). Loss of parkin or PINK1 function increases Drp1-dependent mitochondrial fragmentation. J. Biol. Chem. 284, 22938-22951. doi: 10.1074/jbc.m109.035774

Mortiboys, H., Thomas, K. J., Koopman, W. J. H., Klaffke, S., Abou-Sleiman, P., Olpin, S., et al. (2008). Mitochondrial function and morphology are impaired in parkin-mutant fibroblasts. Ann. Neurol. 64, 555-565. doi: 10.1002/ana.21492

Narendra, D., Kane, L. A., Hauser, D. N., Fearnley, I. M., and Youle, R. J. (2010). p62/SQSTM1 is required for Parkin-induced mitochondrial clustering but not mitophagy; VDAC1 is dispensable for both. Autophagy 6, 1090-1106. doi: 10.4161/auto.6.8.13426

Narendra, D., Tanaka, A., Suen, D. F., and Youle, R. J. (2008). Parkin is recruited selectively to impaired mitochondria and promotes their autophagy. J. Cell Biol. 183, 795-803. doi: 10.1083/jcb.200809125

Nezis, I. P., Lamark, T., Velentzas, A. D., Rusten, T. E., Bjørkøy, G., Johansen, T., et al. (2009). Cell death during Drosophila melanogaster early oogenesis is mediated through autophagy. Autophagy 5, 298-302. doi: 10.4161/auto.5.3.7454

Olzmann, J. A., Li, L., Chudaev, M. V., Chen, J., Perez, F. A., Palmiter, R. D., et al. (2007). Parkin-mediated K63-linked polyubiquitination targets misfolded DJ-1 to aggresomes via binding to HDAC6. J. Cell Biol. 178, 1025-1038. doi: $10.1083 /$ jcb. 200611128

Perciavalle, R. M., Stewart, D. P., Koss, B., Lynch, J., Milasta, S., Bathina, M., et al. (2012). Anti-apoptotic MCL-1 localizes to the mitochondrial matrix and couples mitochondrial fusion to respiration. Nat. Cell Biol. 14, 575-583. doi: $10.1038 /$ ncb2488

Pickrell, A. M., and Youle, R. J. (2015). The roles of PINK1, parkin, and mitochondrial fidelity in Parkinson's disease. Neuron 85, 257-273. doi: 10.1016/j.neuron.2014.12.007

Poole, A. C., Thomas, R. E., Andrews, L. A., McBride, H. M., Whitworth, A. J., and Pallanck, L. J. (2008). The PINK1/Parkin pathway regulates mitochondrial morphology. Proc. Natl. Acad. Sci. U S A 105, 1638-1643. doi: 10.1073/pnas. 0709336105

Poole, A. C., Thomas, R. E., Yu, S., Vincow, E. S., and Pallanck, L. (2010). The mitochondrial fusion-promoting factor mitofusin is a substrate of the PINK1/parkin pathway. PLoS One 5:e10054. doi: 10.1371/journal.pone. 0010054

Rafelski, S. M. (2013). Mitochondrial network morphology: building an integrative, geometrical view. BMC Biol. 11:71. doi: 10.1186/1741-7007-11-71

Rakovic, A., Grünewald, A., Kottwitz, J., Brüggemann, N., Pramstaller, P. P., Lohmann, K., et al. (2011). Mutations in PINK1 and Parkin impair ubiquitination of Mitofusins in human fibroblasts. PLoS One 6:e16746. doi: 10.1371/journal.pone.0016746

Rambold, A. S., Kostelecky, B., Elia, N., and Lippincott-Schwartz, J. (2011). Tubular network formation protects mitochondria from autophagosomal degradation during nutrient starvation. Proc. Natl. Acad. Sci. U S A 108, 10190-10195. doi: 10.1073/pnas.1107402108

Riemensperger, T., Isabel, G., Coulom, H., Neuser, K., Seugnet, L., Kume, K., et al. (2011). Behavioral consequences of dopamine deficiency in the Drosophila central nervous system. Proc. Natl. Acad. Sci. U S A 108, 834-839. doi: 10.1073/pnas.1010930108

Rizzuto, R., Brini, M., Pizzo, P., Murgia, M., and Pozzan, T. (1995). Chimeric green fluorescent protein as a tool for visualizing subcellular organelles in living cells. Curr. Biol. 5, 635-642. doi: 10.1016/s0960-9822(95)00128-x

Rossignol, R., Gilkerson, R., Aggeler, R., Yamagata, K., Remington, S. J., and Capaldi, R. A. (2004). Energy substrate modulates mitochondrial structure and oxidative capacity in cancer cells. Cancer Res. 64, 985-993. doi: 10.1158/00085472.can-03-1101

Sarraf, S. A., Raman, M., Guarani-Pereira, V., Sowa, M. E., Huttlin, E. L., Gygi, S. P., et al. (2013). Landscape of the PARKIN-dependent ubiquitylome in response to mitochondrial depolarization. Nature 496, 372-376. doi: $10.1038 /$ nature 12043
Shpilka, T., Weidberg, H., Pietrokovski, S., and Elazar, Z. (2011). Atg8: an autophagy-related ubiquitin-like protein family. Genome Biol. 12:226. doi: 10.1186/gb-2011-12-7-226

Simonsen, A., Cumming, R. C., Brech, A., Isakson, P., Schubert, D. R., and Finley, K. D. (2008). Promoting basal levels of autophagy in the nervous system enhances longevity and oxidant resistance in adult Drosophila. Autophagy 4, 176-184. doi: 10.4161/auto.5269

Soubannier, V., McLelland, G. L., Zunino, R., Braschi, E., Rippstein, P., Fon, E. A., et al. (2012). A vesicular transport pathway shuttles cargo from mitochondria to lysosomes. Curr. Biol. 22, 135-141. doi: 10.1016/j.cub.2011.11.057

Strappazzon, F., Nazio, F., Corrado, M., Cianfanelli, V., Romagnoli, A., Fimia, G. M., et al. (2015). AMBRA1 is able to induce mitophagy via LC3 binding, regardless of PARKIN and p62/SQSTM1. Cell Death Differ. 22:517. doi: $10.1038 / \mathrm{cdd} .2014 .190$

Strausfeld, N. J., and Hirth, F. (2013). Deep homology of arthropod central complex and vertebrate basal ganglia. Science 340, 157-161. doi: $10.1126 /$ science. 1231828

Tondera, D., Grandemange, S., Jourdain, A., Karbowski, M., Mattenberger, Y., Herzig, S., et al. (2009). SLP-2 is required for stress-induced mitochondrial hyperfusion. EMBO J. 28, 1589-1600. doi: 10.1038/emboj.2009.89

Vincow, E. S., Merrihew, G., Thomas, R. E., Shulman, N. J., Beyer, R. P., MacCoss, M. J., et al. (2013). The PINK1-Parkin pathway promotes both mitophagy and selective respiratory chain turnover in vivo. Proc. Natl. Acad. Sci. US A 110, 6400-6405. doi: 10.1073/pnas.1221132110

Wang, H., Song, P., Du, L., Tian, W., Yue, W., Liu, M., et al. (2011). Parkin ubiquitinates Drp1 for proteasome-dependent degradation: implication of dysregulated mitochondrial dynamics in Parkinson disease. J. Biol. Chem. 286, 11649-11658. doi: 10.1074/jbc.M110.144238

Wang, X., Winter, D., Ashrafi, G., Schlehe, J., Wong, Y. L., Selkoe, D., et al. (2011) PINK1 and Parkin target Miro for phosphorylation and degradation to arrest mitochondrial motility. Cell 147, 893-906. doi: 10.1016/j.cell.2011.10.018

Whitworth, A. J., Theodore, D. A., Greene, J. C., Beneš, H., Wes, P. D., and Pallanck, L. J. (2005). Increased glutathione S-transferase activity rescues dopaminergic neuron loss in a Drosophila model of Parkinson's disease. Proc. Natl. Acad. Sci. U S A 102, 8024-8029. doi: 10.1073/pnas.0501078102

Wong, Y. C., and Holzbaur, E. L. (2014). Optineurin is an autophagy receptor for damaged mitochondria in parkin-mediated mitophagy that is disrupted by an ALS-linked mutation. Proc. Natl. Acad. Sci. U S A 111, E4439-E4448. doi: 10.1073/pnas.1405752111

Yarbrough, D., Wachter, R. M., Kallio, K., Matz, M. V., and Remington, S. J. (2001). Refined crystal structure of DsRed, a red fluorescent protein from coral, at 2.0-A resolution. Proc. Natl. Acad. Sci. U S A 98, 462-467. doi: 10.1073/pnas. 98.2.462

Yoshii, S. R., Kishi, C., Ishihara, N., and Mizushima, N. (2011). Parkin mediates proteasome-dependent protein degradation and rupture of the outer mitochondrial membrane. J. Biol. Chem. 286, 19630-19640. doi: 10.1074/jbc. p110.209338

Ziviani, E., Tao, R. N., and Whitworth, A. J. (2010). Drosophila parkin requires PINK1 for mitochondrial translocation and ubiquitinates mitofusin. Proc. Natl. Acad. Sci. U S A 107, 5018-5023. doi: 10.1073/pnas.0913485107

Conflict of Interest Statement: The authors declare that the research was conducted in the absence of any commercial or financial relationships that could be construed as a potential conflict of interest.

Copyright (C) 2018 Cackovic, Gutierrez-Luke, Call, Juba, O'Brien, Jun and Buhlman. This is an open-access article distributed under the terms of the Creative Commons Attribution License (CC BY). The use, distribution or reproduction in other forums is permitted, provided the original author(s) and the copyright owner are credited and that the original publication in this journal is cited, in accordance with accepted academic practice. No use, distribution or reproduction is permitted which does not comply with these terms. 